\title{
Stimulated Imbalance and the Enhancement of Eddy Kinetic Energy Dissipation by Internal Waves ${ }^{\mathscr{D}}$
}

\author{
ROY BARKAN \\ Department of Atmospheric and Oceanic Science, University of California, Los Angeles, Los Angeles, California \\ KRAIG B. WINTERS \\ Scripps Institution of Oceanography, University of California, San Diego, La Jolla, California \\ JAMES C. MCWILLIAMS \\ Department of Atmospheric and Oceanic Science, University of California, Los Angeles, Los Angeles, California
}

(Manuscript received 12 May 2016, in final form 27 September 2016)

\begin{abstract}
The effects of internal waves (IWs), externally forced by high-frequency wind, on energy pathways are studied in submesoscale-resolving numerical simulations of an idealized wind-driven channel flow. Two processes are examined: the direct extraction of mesoscale energy by externally forced IWs followed by an IW forward energy cascade to dissipation and stimulated imbalance, a mechanism through which externally forced IWs trigger a forward mesoscale to submesoscale energy cascade to dissipation. This study finds that the frequency and wavenumber spectral slopes are shallower in solutions with high-frequency forcing compared to solutions without and that the volume-averaged interior kinetic energy dissipation rate increases tenfold. The ratio between the enhanced dissipation rate and the added high-frequency wind work is 1.3 , demonstrating the significance of the IW-mediated forward cascades. Temporal-scale analysis of energy exchanges among low- (mesoscale), intermediate- (submesoscale), and high-frequency (IW) bands shows a corresponding increase in kinetic energy $E_{k}$ and available potential energy APE transfers from mesoscales to submesoscales (stimulated imbalance) and mesoscales to IWs (direct extraction). Two direct extraction routes are identified: a mesoscale to IW $E_{k}$ transfer and a mesoscale to IW APE transfer followed by an IW APE to IW $E_{k}$ conversion. Spatial-scale analysis of eddy-IW interaction in solutions with high-frequency forcing shows an equivalent increase in forward $E_{k}$ and APE transfers inside both anticyclones and cyclones.
\end{abstract}

\section{Introduction}

Considerable effort has recently been devoted to investigating the routes to dissipation of geostrophic mesoscale eddy kinetic energy (EKE), the largest reservoir of kinetic energy in the ocean (Ferrari and Wunsch 2009). The focus has been on identifying and quantifying possible energy transfer mechanisms from the balanced geostrophic eddy field to unbalanced ageostrophic motions, and the subsequent forward energy cascade to

Supplemental information related to this paper is available at the Journals Online website: http://dx.doi.org/10.1175/ JPO-D-16-0117.s1.

Corresponding authore-mail: Roy Barkan, rbarkan@atmos. ucla.edu dissipation. Potential transfer mechanisms include spontaneous imbalance via submesoscale instabilities (Molemaker et al. 2005; Capet et al. 2008; Molemaker et al. 2010) that are predominantly confined to the surface boundary layer (SBL; D'Asaro et al. 2011; McWilliams 2016), spontaneous emission of internal waves (IWs; Vanneste 2013), wave capture (Bühler and McIntyre 2003, 2005), and wave excitation due to deep geostrophic flow encountering rough topography (Nikurashin et al. 2013).

Several studies have also examined different mechanisms through which externally forced near-inertial IWs (NIWs) can extract energy from the balanced flow. Xie and Vanneste (2015, hereinafter XV15) and Wagner and Young (2016) developed an asymptotic theory for the interaction between low Rossby number quasigeostrophic (QG) flow and NIWs. They derived energy and action 
conservation laws and illustrated a mechanism through which NIWs can directly extract energy from the balanced QG flow. Using mesoscale eddy-permitting numerical simulations of wind-forced channel flow, Taylor and Straub (2016) demonstrated that near-inertial Reynolds stresses, associated with forced NIWs, can provide a kinetic energy sink for mesoscale geostrophic motions. Focusing on submesoscale processes with Rossby number of $O(1)$, Thomas (2012) investigated the effects of frontogenetic strain on IWs in an idealized, two-dimensional model and suggested that IWs can extract energy from a geostrophically balanced deformation field and transfer it to ageostrophic motion, thereby catalyzing the forward energy cascade. Later, Whitt and Thomas (2015, hereinafter WT15) employed a slab mixed layer model and twodimensional numerical simulations to demonstrate that inertial oscillations can indeed extract energy from a unidirectional, laterally sheared geostrophic current. Finally, Grisouard and Thomas (2015) showed how upwardpropagating IWs experience critical reflection against the ocean surface in a geostrophically balanced front and, subject to viscous effects, can exchange energy with the front eventually leading to its dissipation.

In the present study, we use three-dimensional, submesoscale-resolving numerical simulations of windforced energetic mesoscale and submesoscale flow as well as IWs in order to examine the mechanisms leading to the enhancement of EKE dissipation by externally forced IWs, either near the surface or in the interior. We aim to distinguish between direct IW extraction mechanisms followed by an IW forward cascade to dissipation and stimulated imbalance, a process through which externally forced IWs stimulate the forward mesoscale to submesoscale energy cascade to dissipation. We choose a channel configuration, centered at high latitudes, similar to the Antarctic Circumpolar Current (ACC). Previous studies of energy transfers in the ACC suggest that topographically generated internal waves (Nikurashin et al. 2013) as well as submesoscale instabilities (Barkan et al. 2015) can provide important pathways to dissipation for EKE. Both studies, however, considered steady wind forcing only, thereby excluding wind generated IWs and thus ignoring any possible interactions. We show that externally forced IWs enhance EKE dissipation and catalyze energy transfer from low-frequency (LF), large-scale motions. The ratio of the enhanced EKE dissipation to the IW wind work in our solutions is about 1.3, demonstrating the efficiency of the IW-mediated routes to dissipation.

\section{Experimental setup}

We consider an idealized channel flow on an $f$ plane configured to resemble the dynamics of the ACC (although without loss of generality we take $f>0$ ). The zonal, meridional, and vertical $(\hat{x}, \hat{y}, \hat{z})$ dimensions are $L_{x}=L_{y}=200 \mathrm{~km}$, and $H=2000 \mathrm{~m}$ and the associated equally spaced resolutions are $d x=d y=390 \mathrm{~m}$, and $d z=$ $15 \mathrm{~m}$. The corresponding velocity vector $\mathbf{u}=(u, v, w)$, and buoyancy $b$ is the only active tracer for the assumed linear equation of state. The boundary conditions are periodic in the zonal direction, free-slip walls in the meridional direction, and free-slip rigid lid in the vertical. The initial stratification profile is based on observations from the Southern Ocean (Garabato et al. 2004) with buoyancy frequency $N$ ranging from a few times the Coriolis frequency $f$ in the bottom $1000 \mathrm{~m}$ to $20 f$ at $200-\mathrm{m}$ depth and then gradually decaying toward the surface, with $f=1.2 \times 10^{-4} \mathrm{~s}^{-1}$. Our analysis is based on three numerical simulations of the nonhydrostatic Boussinesq equations of motion utilizing the pseudospectral model flow_solve (Winters and de la Fuente 2012). We add eighth-order horizontal "hyperdiffusion" terms and fourth-order vertical hyperdiffusion terms to the momentum and buoyancy equations to remove energy as close as possible to the grid scale, thereby increasing the "effective" resolution of inviscid dynamics. Linear bottom drag is applied as a body force described in (A.1a) and (A.6) with a drag coefficient $r=1 \times 10^{-7} \mathrm{~s}^{-1}$.

The first simulation, hereinafter referred to as lowfrequency wind (LFW), is forced by a jetlike wind stress $\tau_{s}$ of the form

$$
\tau_{s}(y)=\tau_{0} \sin ^{2}\left(\frac{\pi y}{L_{y}}\right) \hat{\mathbf{x}},
$$

where $\rho_{0} \tau_{0}=0.1 \mathrm{~N} \mathrm{~m}^{-2}$, and $\hat{\mathbf{x}}$ is the unit vector in the zonal direction. This steady wind forcing drives an eastward zonal jet and the associated Ekman pumping $w_{\mathrm{Ek}}=-f^{-1} d \tau_{s} / d y$ corresponds to downwelling near $y=$ $L_{y} / 4$ and upwelling near $y=3 L_{y} / 4$. The Ekman circulation tilts isopycnals and consequently generates baroclinic instability that forms mesoscale, baroclinic eddies.

The second simulation, hereinafter referred to as high-frequency wind (HFW), is forced by a stochastic wind stress $\tau_{w}$ of the form

$$
\tau_{w}(x, t)=\tau_{0} F(x) W(t) \hat{\mathbf{x}},
$$

where $F(x)=\cos \left(2 \pi x / L_{x}\right)$, and $W(t)$ is a Gaussian white noise function with zero mean and variance $\eta^{2}$ that varies over $\Delta t=3 d t=1 \mathrm{~min}$, with $d t$ being the model time step. These rapid variations in $W(t)$ ensure that it adequately represents a Markovian white noise process while maintaining numerical stability. The $x$ dependence in $F(x)$ sets the scale of the excited internal waves to be $O\left(L_{x}\right)$, while ensuring a zero zonal-mean 


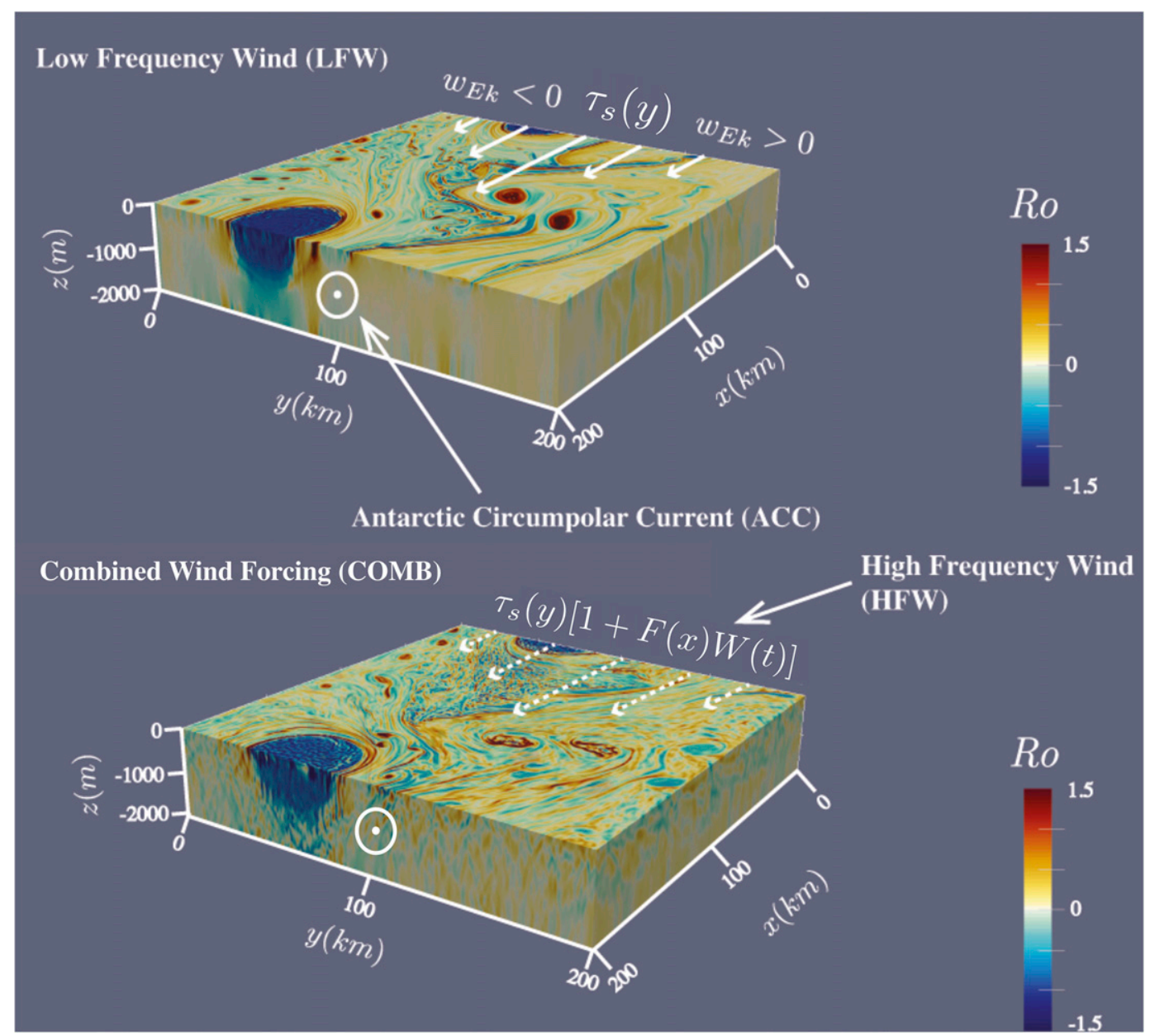

FIG. 1. A schematic illustrating the experimental setup discussed in section 2. The underlying plotted field is the Rossby number Ro $=\zeta / f$, where $\zeta$ is the vertical component of vorticity, and $f$ is the Coriolis frequency.

wind stress. The stochastic forcing is a simple model for high-frequency (HF) wind that efficiently excites IWs.

The third simulation, hereinafter referred to as COMB, is forced by a combined wind stress $\tau_{\text {total }}$ of the form

$$
\tau_{\text {total }}(x, y, t)=\tau_{s}\left(1+\frac{\tau_{w}}{\tau_{0}}\right),
$$

which has the same time and/or zonal mean as (1). Figure 1 shows a schematic of the experimental setup. Tidal- and topographically generated IWs are deliberately excluded to focus in particular on the interactions among surface-forced mesoscales, submesoscales, and internal waves. Furthermore, IWs are allowed to reflect back from the meridional walls to mimic other wave sources outside of the domain. Finally, the wind stress in all cases is applied as a body force [(A.4)] that is confined to the upper $\sim 80 \mathrm{~m}$ and is transmitted by a combination of Laplacian viscosity and hyperviscosity. Additional information about the numerical simulations and setup are provided in appendix A.

The LFW simulation is run for 4 months starting from a lower-resolution equilibrated solution until a steady state in the volume-averaged kinetic energy equation is reached. The COMB solution is run for 4 months starting from the equilibrated high-resolution LFW solution and the HFW solution is run for 4 months starting from rest. In all cases only the last 3 months are analyzed. Figure 2 shows the initial stratification profile for all solutions, and the time- and zonal-averaged perturbation density and zonal velocity from the COMB solution.

\section{Overview of results, comparison with observations}

Representative snapshots of horizontal slices of the Rossby number Ro, the vertical component of relative vorticity normalized by $f$, at the surface and vertical 

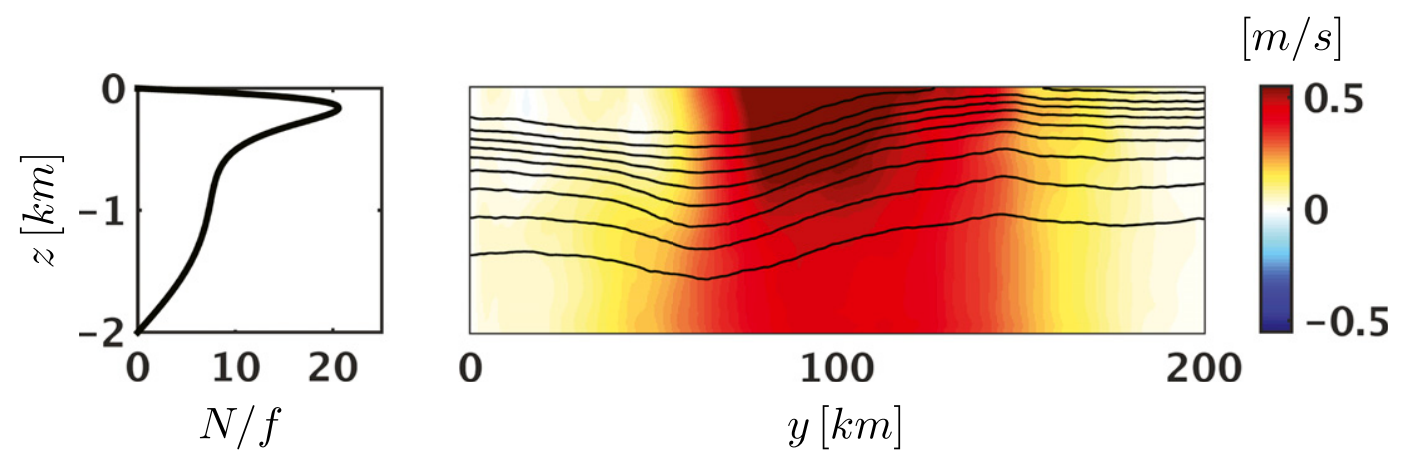

FIG. 2. (left) The initial stratification profile for the three numerical solutions. (right) The zonally averaged zonal velocity (color) and perturbation density field (contour lines). Isopycnal interval is $0.04 \mathrm{~kg} \mathrm{~m}^{-3}$ starting from $27.58 \mathrm{~kg} \mathrm{~m}^{-3}$ (bottommost contour).

velocity $w$ at $z=-150 \mathrm{~m}$ are shown in Fig. 3. The elongated zonal structures of both fields in the HFW solution are IWs that propagate northward and southward throughout the domain. The Rossby numbers are small compared with the LFW and COMB solutions. In both the LFW and COMB solutions, there is one large anticyclone and two midsize cyclones along with smaller eddies, fronts, and filaments. These flow features have Ro $\sim O(1)$, evidence of ageostrophic dynamics across a range of spatial scales. The COMB solution exhibits smaller-scale features both within and between the larger eddies compared with the LFW solution, a first indication for the interactions among mesoscales, submesoscales, and IWs. The vertical velocity field shows that smaller scales are already apparent within the cyclones and anticyclones in the LFW solution. However, in the COMB solution the magnitude of the vertical velocity is larger than the sum of HFW and LFW (bottom right).

Snapshots of the small-scale kinetic energy dissipation rate [(5)] for the LFW and COMB solutions are shown in Fig. 4. In LFW most of the dissipation is observed in the anticyclone and midsize cyclones, suggesting that without HF forcing a kinetic energy transfer to smaller scales is predominantly active in these coherent structures. In
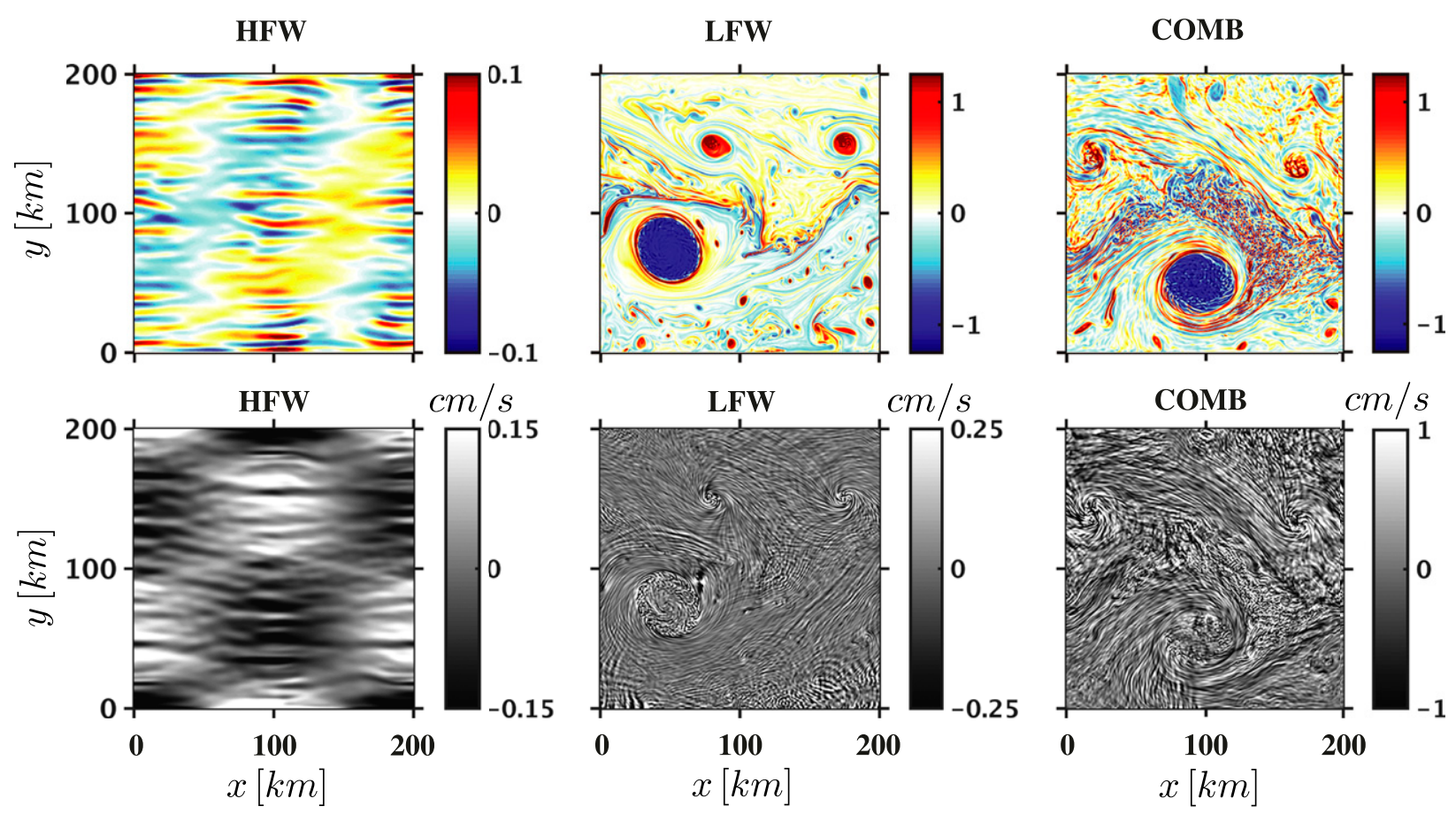

FIG. 3. Snapshots of horizontal slices of Rossby number, vertical vorticity normalized by the (top) Coriolis frequency at the surface and (bottom) vertical velocity at 150-m depth for (left) HFW, (center) LFW, and (right) COMB solutions. Note the different colorbar ranges. 


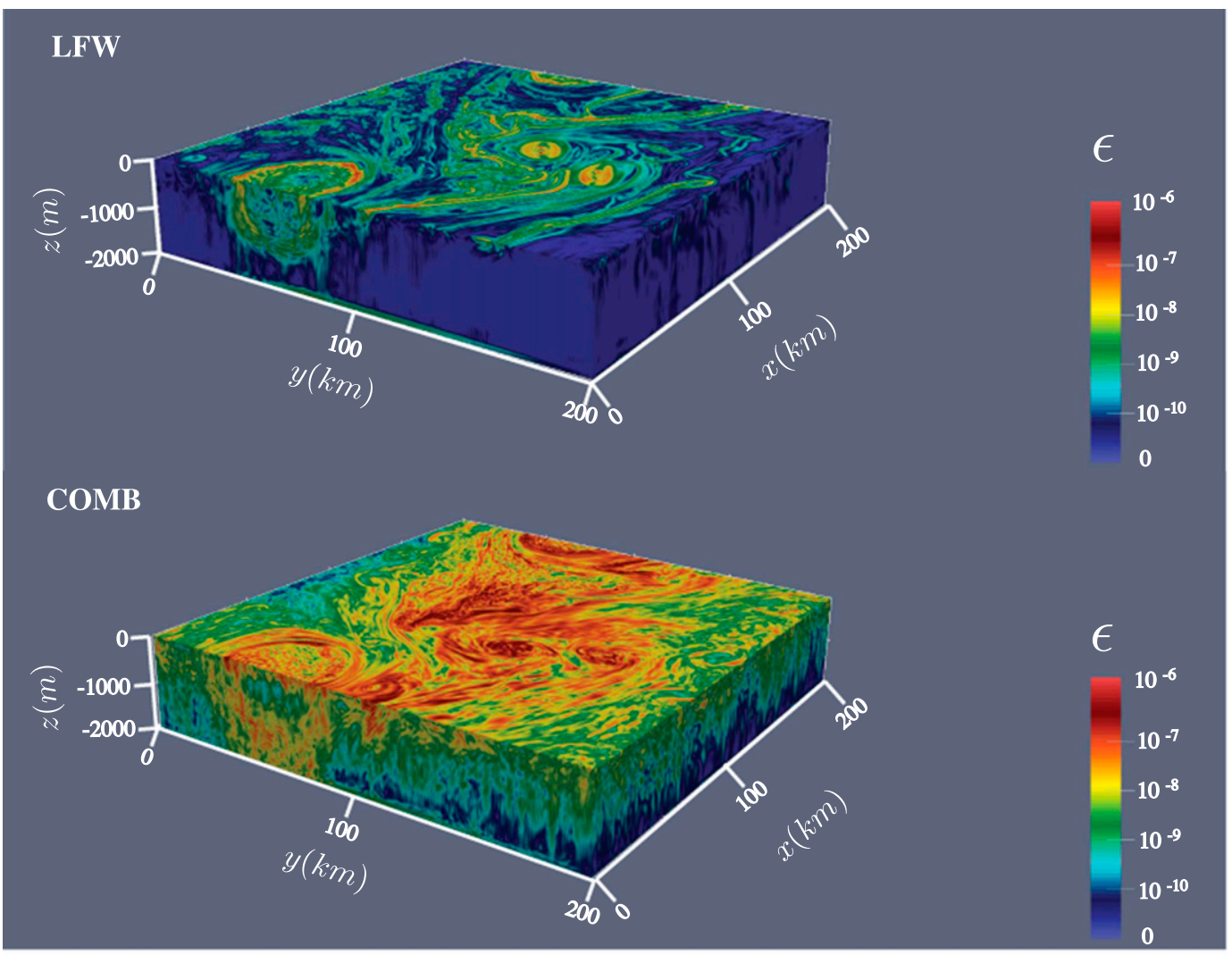

FIG. 4. Snapshots of small-scale kinetic energy dissipation [(5); $\left.\mathrm{W} \mathrm{kg}^{-1}\right]$ for the (top) LFW and (bottom) COMB solutions.

$\mathrm{COMB}$, one can observe an overall enhancement in dissipation at the surface in the vicinity of frontal regions as well as in the interior of the anticyclonic and cyclonic eddies.

The frequency and wavenumber spectra of the horizontal velocity at $z=-150$ and $-1000 \mathrm{~m}$ for the three solutions are shown in Fig. 5. The energy fraction in the IW frequency band in the HFW solution (brown curve, Fig. 5a) contains $\sim 90 \%$ of the total energy. The energy fraction in the frequency band between $f$ and $1.1 f$ (nearinertial frequency band) contains $\sim 77 \%$ of the total energy. This is a good indication that although the stochastic forcing [(2)] acts on all frequencies it predominantly excites IWs. Comparing the LFW and COMB solutions (Figs. 5a,b) shows that the frequency spectral slopes are shallower for COMB at both depths. The COMB spectral slope at $z=-1000 \mathrm{~m}$ (blue) matches observations from the Southern Ocean (Phillips and Rintoul 2000; Ferrari and Wunsch 2009, their Fig. 2b) in both sub- and superinertial frequencies as well as the generic Garrett-Munk (GM) spectrum (Garrett and Munk 1972) in the IW band (dashed magenta line in Fig. 5b). Similarly, the wavenumber spectral slope at $1000-\mathrm{m}$ depth in the COMB solution is in good agreement with that of the GM spectra (dashed magenta line in Fig. 5d). In addition, the wavenumber spectral slopes in the COMB solution are much shallower than in the LFW solution at both depths (Figs. $5 \mathrm{c}, \mathrm{d})$, illustrating that more energy is found at smaller scales in the COMB solution, as suggested by Figs. 3 and 4. Figure 6 shows that the COMB energy content in the near- to superinertial frequency band is larger by an order of magnitude than that associated with the linear superposition of the HFW and LFW solutions. Furthermore, the similar energy content in LFW and COMB at lower frequencies illustrates that the amount of LF energy that is injected by the HF forcing [(2)] is negligible. Because the wavenumber spectral slope of both the HFW and LFW solutions is steeper than that of the COMB solution (Figs. $5 c$,d), it is evident that the energy levels of the latter are higher than the linear superposition of the first two.

\section{Energetics}

\section{a. Volume-averaged kinetic energy}

The volume-averaged kinetic energy equation for our model takes the form 

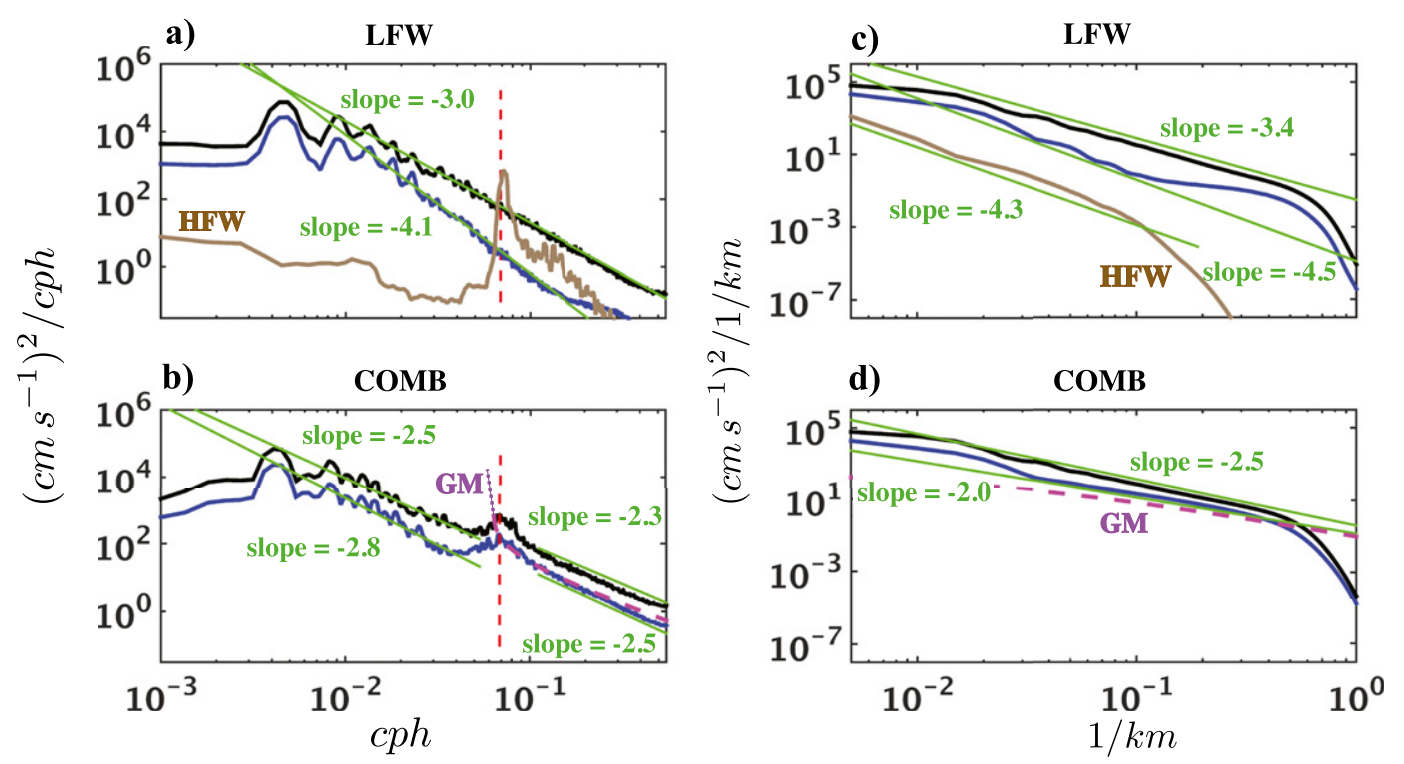

FIG. 5. (a),(b) Horizontal velocity frequency spectra and (c),(d) meridionally averaged wavenumber spectra at $z=-150 \mathrm{~m}$ (black) and $z=-1000 \mathrm{~m}$ (blue). (a) and (c) are for LFW, and (b) and (d) are for COMB. Brown lines in (a) and (c) show the HFW spectra at $z=-150 \mathrm{~m}$. The dashed magenta line in (b) and (d) corresponds to the Garret Munk spectrum. Dashed red lines denote the inertial frequency.

$$
\frac{d\left\langle E_{k}\right\rangle}{d t}=\langle w b\rangle+\tau_{\text {work }}-\epsilon_{d}-\epsilon
$$

where $E_{k}=0.5 \mathbf{u} \cdot \mathbf{u},\langle\rangle$ denotes the volume average, $\langle w b\rangle$ is the reversible conversion between kinetic and available potential energies (APE), and the wind work $\tau_{\text {work }}$ and bottom drag dissipation $\epsilon_{d}$ are defined in (A.5) and (A.6), respectively. The dissipation at small scales

$\epsilon=\nu\left\langle\frac{\partial u_{i}}{\partial x_{k}} \frac{\partial u_{i}}{\partial x_{k}}\right\rangle+\nu_{h}^{*}\left\langle\frac{\partial^{4} u_{i}}{\partial x_{j}^{4}} \frac{\partial^{4} u_{i}}{\partial x_{j}^{4}}\right\rangle+\nu_{v}^{*}\left\langle\frac{\partial^{2} u_{i}}{\partial x_{3}^{2}} \frac{\partial^{2} u_{i}}{\partial x_{3}^{2}}\right\rangle$,

where $i, k=1-3, j=1,2$, and summation over repeated indices is assumed. The quantity $\nu$ is the viscosity and $\nu_{h}^{*}, \nu_{v}^{*}$ are the horizontal and vertical hyperviscosities, respectively. See appendix A for further detail. In all of our results the second and third terms on the right-hand side of (5) account for $\sim 96 \%$ of the total dissipation. We refer to the dissipation associated with the first term for $k=3$ and the last term as vertical dissipation and the remaining terms as horizontal dissipation.

In what follows, we distinguish the eddy contributions to $\epsilon, \varepsilon_{d},\langle w b\rangle$, where eddy components, denoted by primes, are defined as perturbation from a zonal mean ( ). Consequently, the EKE dissipation terms $\epsilon_{d}^{\prime}$ and $\epsilon^{\prime}$ are identical to (A.6) and (5) respectively, with $u_{i}$ replaced by $u_{i}^{\prime}$. The potential to kinetic energy conversion term is decomposed via $\langle w b\rangle=\langle\bar{w} \bar{b}\rangle+\left\langle\overline{w^{\prime} b^{\prime}}\right\rangle$. Throughout the paper, volume-averaged energy tendency units are converted from watts per kilogram to milliwatts per square meter.

The terms in (4), computed for the LFW and COMB solutions, are shown in Fig. 7. In the LFW case almost all of the wind work is dissipated via bottom drag (Fig. 7a). The eddy decomposition (Fig. 7c) shows that most of the energy dissipated by bottom drag is associated with the mean flow $(\sim 90 \%)$. This is an expected result for a flat-bottom channel configuration, where the mean ACC is unrealistically strong (Munk and Palmén 1951; Tréguier and McWilliams 1990). The eddy conversion term $\left\langle\overline{w^{\prime} b^{\prime}}\right\rangle>0$ is larger in magnitude and has the opposite sign compared with the total $\langle w b\rangle$ because, in this configuration, the buildup of APE by the Ekman-driven mean flow $(\langle\bar{w} \bar{b}\rangle<0$, not shown $)$ is compensated by APE release through baroclinic instability. The EKE volume-averaged vertical dissipation is $0.1 \pm 0.0 \mathrm{~mW} \mathrm{~m}^{-2}$, and the ratio between the vertical to the horizontal dissipation is 0.5 .

In the COMB solution (Figs. 7b,d), $\epsilon_{d}^{\prime}$ remains largely unchanged, whereas $\epsilon^{\prime} \approx \epsilon$ increases by $\sim 3.9 \mathrm{~mW} \mathrm{~m}^{-2}$. The added HF wind work of $3 \mathrm{~mW} \mathrm{~m}^{-2}$ might be expected to dissipate at small scales; however, the excess $\sim 0.9 \mathrm{~mW} \mathrm{~m}^{-2}$ can only be transferred from LF motions, implying an enhanced forward energy cascade; $\left\langle\overline{w^{\prime} b^{\prime}}\right\rangle$ is increased by $\sim 0.7 \mathrm{~mW} \mathrm{~m}^{-2}$, and $\langle\bar{w} \bar{b}\rangle$ is decreased by $\sim 0.5 \mathrm{~mW} \mathrm{~m}^{-2}$ in the COMB solution (becoming more negative). This implies that IWs amplify the extraction of APE from the wind forcing as well as the conversion rate to EKE. The vertical EKE dissipation now accounts 


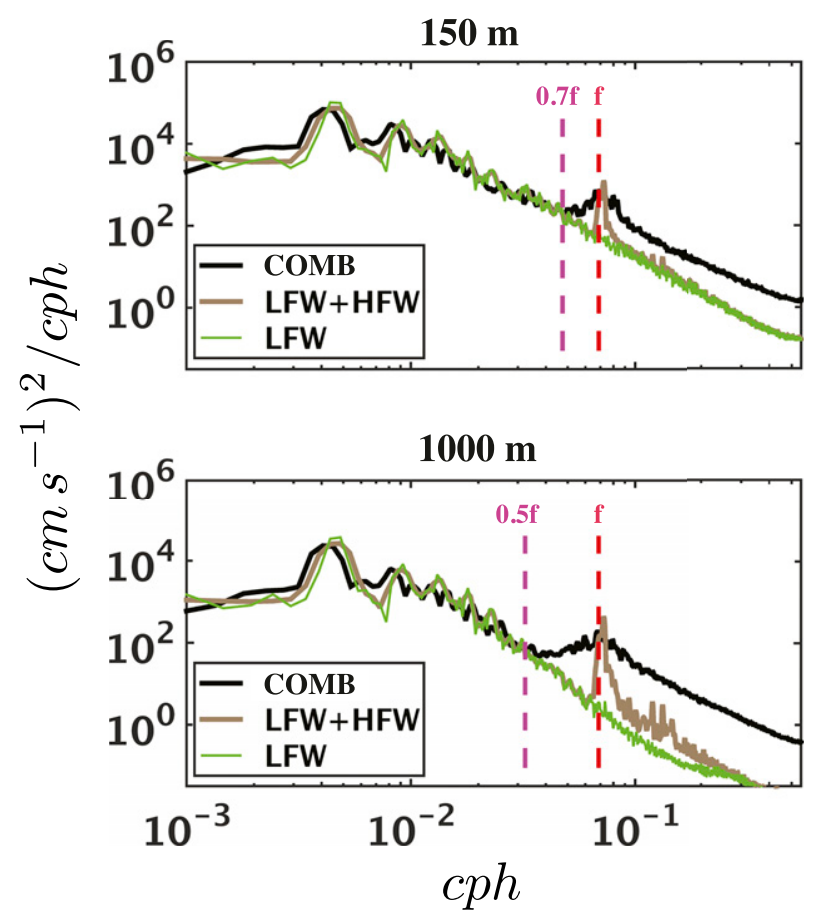

FIG. 6. Horizontal velocity frequency spectra of the LFW solution, COMB solution, and HFW + LFW solutions. Compare with Fig. 5. The values of the LFW and HFW + LFW spectra in the superinertial frequency range of the top panel are similar.

for $2.0 \pm 0.0 \mathrm{~mW} \mathrm{~m}^{-2}$ of the total dissipation, and the ratio between the vertical to the horizontal dissipation increases to $\approx 1$. This suggests an enhancement in the vertical shear in the COMB solution.

\section{b. Low-frequency/high-frequency energy conversions}

To better understand the way in which externally forced IWs amplify the EKE dissipation in the solution, we develop a framework to analyze energy exchanges across temporal scales. To quantify the rate of LF to HF energy exchanges, we decompose any field $\phi$ via

$$
\phi=\tilde{\phi}+\hat{\phi},
$$

where $\widetilde{()}$ denotes a filter operator and, in general,

$$
\begin{aligned}
& \tilde{\hat{\phi}} \neq 0, \quad \text { and } \\
& \tilde{\tilde{\phi}} \neq \tilde{\phi},
\end{aligned}
$$

so that (6) defines a Leonard decomposition (Leonard 1974). ${ }^{1}$ The LF and HF kinetic energies are thus defined as

\footnotetext{
${ }^{1}$ It is assumed that $\widetilde{\partial \phi} / \partial x_{i}=\partial \tilde{\phi} / \partial x_{i}$ and that $\widetilde{\partial \phi} / \partial t=\partial \tilde{\phi} / \partial t$, which is sensible for the Gaussian filter we use in the analysis.
}

$$
\begin{aligned}
E_{k_{\mathrm{LF}}} & =\frac{1}{2} \widetilde{u}_{i} \widetilde{u}_{i} \text {, and } \\
E_{k_{\mathrm{HF}}} & =\frac{1}{2}\left(\widetilde{u_{i} u_{i}}-\widetilde{u}_{i} \widetilde{u}_{i}\right) .
\end{aligned}
$$

In appendix $\mathrm{B}$, we show the inviscid evolution equations for $E_{k_{\mathrm{HF}}}$ and the high-frequency $\mathrm{APE}\left(\mathrm{APE}_{\mathrm{HF}}\right)$ to be

$$
\begin{gathered}
\frac{D}{D t} E_{k_{\mathrm{HF}}}+\frac{\partial}{\partial x_{k}}\left(\mathrm{LSW}_{k}+\mathrm{PW}_{k}\right)=\mathrm{BP}+\mathrm{SP}, \text { and } \\
\frac{D}{D t} \mathrm{APE}_{\mathrm{HF}}+\frac{\partial}{\partial x_{k}}\left(\mathrm{APEW}_{k}-\tilde{b} \frac{B_{k}}{N^{2}}\right) \\
\quad+\frac{\partial}{\partial z} \mathcal{N}^{2}\left(\tilde{b} B_{3}-\mathrm{BVW}_{3}\right)=-\mathrm{BP}+\mathrm{APEP}
\end{gathered}
$$

where $i, k=1-3, D / D t=\partial / \partial t+\tilde{u}_{k} \partial / \partial x_{k}, \quad \mathrm{APE}_{\mathrm{HF}}=$ $1 / 2\left(\widetilde{b^{2}} / N^{2}-\tilde{b}^{2} / N^{2}\right)$, and $N^{2}(z)$ is the time- and horizontally averaged stratification. Note that this form of APE is an approximation to the more general definition (Winters et al. 1995). The HF APE to HF $E_{k}$ buoyancy conversion term

$$
\mathrm{BP} \equiv \widetilde{w b}-\tilde{w} \tilde{b}
$$

the LF to HF shear production term

$$
\mathrm{SP} \equiv-\tau_{i k}^{r} \frac{\partial \tilde{u}_{i}}{\partial x_{k}}
$$

and the LF to HF APE production term

$$
\mathrm{APEP} \equiv-\frac{B_{k}}{N^{2}} \frac{\partial b}{\partial x_{k}}\left(\delta_{k 1}+\delta_{k 2}\right),
$$

where

$$
\begin{aligned}
\tau_{i k}^{r} & \equiv \widetilde{u_{i} u_{k}}-\tilde{u}_{i} \tilde{u}_{k}, \quad \text { and } \\
B_{k} & \equiv \widetilde{u_{k} b}-\tilde{u}_{k} \tilde{b},
\end{aligned}
$$

$\delta_{k 1}, \delta_{k 2}$ are Kronecker deltas, and the rest of the terms in (8) are described in appendix B. Positive SP and APEP indicate LF to HF transfers of kinetic and available potential energies, respectively. Positive BP indicates a conversion from $\mathrm{APE}_{\mathrm{HF}}$ to $E_{k_{\mathrm{HF}}}$. Note that aside from the different decompositions (Leonard vs Reynolds), SP differs from the "advective sink" in Taylor and Straub (2016) by a term corresponding to the divergence of a flux. The two quantities will have the same volume integral but different spatial structures. It is only SP that appears with opposite signs in (B.5) and (8a) and is thus the only "true" exchange term.

The quantity BP corresponds to energy conversion by the vertical velocity $w$, and APEP corresponds to energy 

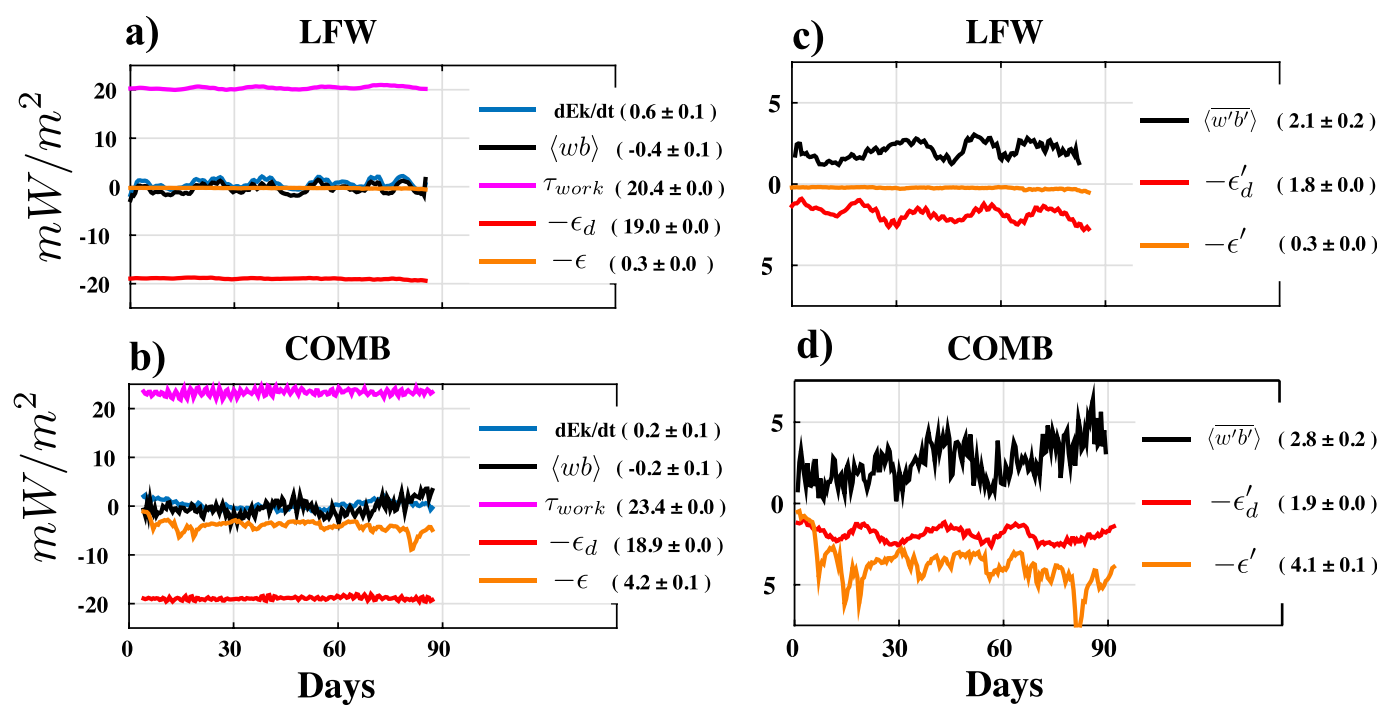

FIG. 7. Volume-averaged terms in the $E_{k}$ evolution equation [(4); $\mathrm{mW} \mathrm{m}^{-2}$ ] for the (a),(c) LFW solution and (b),(d) COMB solution. (a) and (b) display total quantities while (c) and (d) display eddy quantities. Values in the legend denote time-averaged quantities and standard errors.

transfer by the horizontal velocity $(u, v)$. For completeness, we decompose SP into its horizontal component

$$
\mathrm{SP}_{\mathrm{hor}} \equiv-\tau_{i k}^{r} \frac{\partial \tilde{u}_{i}}{\partial x_{k}}\left(\delta_{k 1}+\delta_{k 2}\right)
$$

and vertical component

$$
\mathrm{SP}_{\mathrm{ver}} \equiv-\tau_{i 3}^{r} \frac{\partial \tilde{u}_{i}}{\partial z}
$$

Using (9), (10), and (11), we aim to quantify energy transfers between the different temporal scales in the solutions and distinguish between transfers from low to intermediate frequencies and transfers from low to high frequencies. To this end, we apply Gaussian filters with two filter widths: $\mathcal{G}_{0.3}$, which has a filter width of $0.3 f$ (equivalent to $48 \mathrm{~h}$ ), and $\mathcal{G}_{0.9}$, which has a filter width of $0.9 f$ (equivalent to $16 \mathrm{~h}$ ). The frequency spectra associated with the low-pass horizontal velocities $\tilde{\mathbf{u}}_{h}$ and highpass horizontal velocities $\hat{\mathbf{u}}_{h}$ for the two filter widths are shown in Fig. 8. For $\mathcal{G}_{0.3}$, the low-pass velocity spectra (solid cyan) accounts for motions with time scales longer than $\sim 8$ days and high-pass velocity spectra (dashed cyan) accounts for motions with time scales shorter than $\sim 4$ days. For $\mathcal{G}_{0.9}$, the low-pass velocity spectra (solid green) accounts for motions with time scales longer than $\sim 3$ days and high-pass velocity spectra (dashed green) accounts for motions with time scales shorter than $\sim 1$ day, which include the entire inertial peak. The intermediate frequency band, corresponding to the spectra associated with the difference between the high-pass velocities (Fig. 8c), spans time scales between $\sim 2$ and 4 days. In what follows we will associate low-, intermediate-, and high-frequency motions with mesoscales, submesoscales, and IWs, respectively. Energy transfer from mesoscale to IW frequencies will be referred to as direct extraction, and energy transfer from mesoscale to submesoscale frequencies will be referred to as stimulated imbalance. Evidently, in these high Ro solutions there is no clear spectral gap between mesoscales and submesoscales and submesoscales and IWs. Furthermore, IWs can be Doppler shifted by lower-frequency motions over a range that can be estimated based on the width of the inertial peak in the COMB solution compared with the HFW solution (Fig. 6). Finally, the Gaussian filters used are imperfect and always allow some "leakage" of energy between the different frequency bands. To evaluate the validity of the filtering approach we also compute the transfer terms using a $\mathcal{G}_{2.4}$ filter, which has a width of $2.4 f$ (equivalent to $6 \mathrm{~h}$ ). For this filter, high-pass horizontal velocities account for motions with frequencies larger than $1.25 f$ (i.e., well within the IW frequency band), and the intermediate $\mathcal{G}_{0.9}-\mathcal{G}_{2.4}$ frequency band accounts for motions with time scales of $\sim 18-36 \mathrm{~h}$ or 1.3 inertial periods to 1.5 days (not shown).

The time- and volume-averaged shear production (SP), APE production (APEP), and buoyancy conversion (BP) for the LFW and COMB solutions are shown in Table 1. For all filter widths, SP and APEP increase in the COMB solution relative to the LFW solution, indicating an enhanced LF to HF kinetic and APE energy transfer. BP is decreased in the COMB 
a)

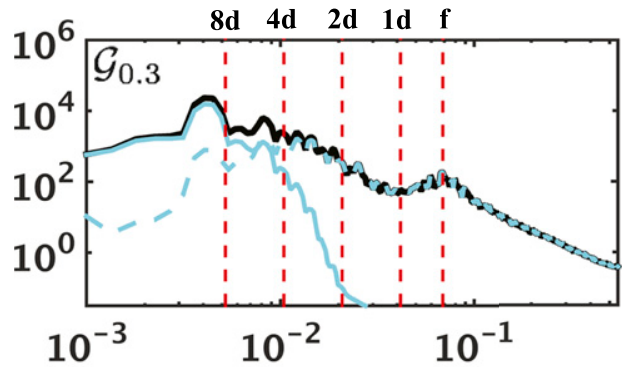

b)

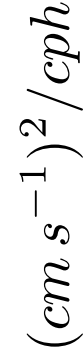

c)
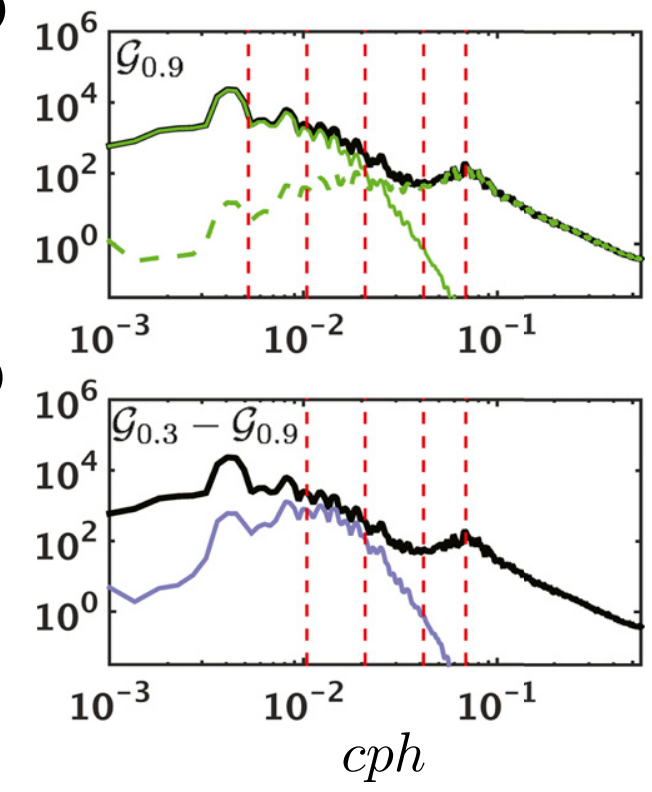

FIG. 8. Frequency spectra of the horizontal unfiltered velocity (solid black), the (a) $\mathcal{G}_{0.3}$ low-pass (solid cyan) and high-pass (dashed cyan) horizontal velocity, and the (b) $\mathcal{G}_{0.9}$ low-pass (solid green) and high-pass (dashed green) horizontal velocity. (c) Frequency spectra of the difference between $\mathcal{G}_{0.3}$ and $\mathcal{G}_{0.9}$ high-pass velocities (light blue). Dashed red lines denote $1 / 8,1 / 4,1 / 2$, and 1 day $^{-1}$, and inertial frequencies. COMB solution at $1000-\mathrm{m}$ depth.

solution for the $\mathcal{G}_{0.3}$ and $\mathcal{G}_{0.9}$ filters indicating a reduction in the HF APE to HF $E_{k}$ conversion. In the LFW solution, the magnitudes of SP and APEP remain relatively unchanged for the $\mathcal{G}_{0.3}$ and $\mathcal{G}_{0.9}$ filters and are substantially reduced for the $\mathcal{G}_{2.4}$ filter. This indicates that little energy is transferred to frequencies larger than $1.25 f$, suggesting that the IW energy in this solution is small. In the COMB solution, the magnitudes of SP and APEP for the $\mathcal{G}_{0.9}$ and $\mathcal{G}_{2.4}$ filters are similar and smaller than that of $\mathcal{G}_{0.3}$, suggesting that a considerable fraction of the energy remains at submesoscale time scales and is not transferred all the way to IW time scales. It is this fraction of the energy and its associated dissipation that we attribute to stimulated imbalance. The fact that one could infer that a considerable fraction of the energy remains at intermediate (submesoscale) time scales irrespective of whether the $\mathcal{G}_{0.9}$ or $\mathcal{G}_{2.4}$ filters are used indicates that the distinction between direct extraction and stimulated imbalance is robust. We choose the $\mathcal{G}_{0.9}$ filter to distinguish IWs because it contains the entire inertial peak and plausibly more IW energy (Fig. 8b). Note that SP for the $\mathcal{G}_{2.4}$ filter in the COMB solution is larger than that of $\mathcal{G}_{0.9}$. This is possibly associated with the large fraction of APE that is converted to $E_{k}$ at time scales of $\sim 18-36 \mathrm{~h}$ (the $\mathcal{G}_{0.9}-\mathcal{G}_{2.4}$ frequency band).

To examine the vertical structure of the conversion terms we compute the horizontal- and time-averaged values of SP, APEP, and BP (Fig. 9). There is a substantial increase in $\mathrm{SP}_{\text {ver }}[(14)]$ of the COMB solution in the upper ocean (Fig. 9a) for both filter widths. This indicates that a substantial fraction of the $E_{k}$ that is transferred to the IW frequency band is associated with $\mathrm{SP}_{\mathrm{ver}}$. This is in agreement with the increased ratio of vertical to horizontal EKE dissipation discussed in section 4a. On the contrary, there is significant increase in $\mathrm{SP}_{\text {hor }}$ of the COMB solution in the upper ocean for the $\mathcal{G}_{0.3}$ filter width, whereas $\mathrm{SP}_{\text {hor }}$ for the $\mathcal{G}_{0.9}$ filter width has very similar values to those of the LFW solution. This suggests that most of the $E_{k}$ transferred because of $\mathrm{SP}_{\text {hor }}$ is to the submesoscale frequency band. BP in the COMB solution is reduced with respect to LFW for both filter widths in the upper ocean with a considerable fraction of the HF APE converted to HF $E_{k}$ in the IW frequency band. APEP in the COMB solution is significantly larger in the upper ocean for the $\mathcal{G}_{0.3}$ filter width and close to the bottom for the $\mathcal{G}_{0.9}$ filter width. This implies that LF APE is transferred to the submesoscale frequency band in the upper ocean and to the IW frequency band near the bottom. To emphasize the kinetic and available potential energy transfers to the IW frequency band, we compute the time- and zonal-averaged

TABLE 1. Time- and volume-averaged HF to LF SP [(10)], HF APE to HF $E_{k}$ BP [(9)], and HF to LF APEP [(11)] (mW m $\left.{ }^{-2}\right)$ computed using Gaussian filters with filter widths of $0.3 f\left(\mathcal{G}_{0.3}\right), 0.9 f\left(\mathcal{G}_{0.9}\right)$, and $2.4 f\left(\mathcal{G}_{2.4}\right)$.

\begin{tabular}{ccccccc}
\hline \hline Conversion term & LFW $\mathcal{G}_{0.3}$ & COMB $\mathcal{G}_{0.3}$ & LFW $\mathcal{G}_{0.9}$ & COMB $\mathcal{G}_{0.9}$ & LFW $\mathcal{G}_{2.4}$ & COMB $\mathcal{G}_{2.4}$ \\
\hline SP & $0.31 \pm 0.02$ & $1.82 \pm 0.02$ & $0.30 \pm 0.05$ & $0.85 \pm 0.03$ & $0.04 \pm 0.02$ & $1.10 \pm 0.03$ \\
BP & $1.87 \pm 0.01$ & $1.37 \pm 0.01$ & $1.29 \pm 0.01$ & $1.16 \pm 0.01$ & $0.48 \pm 0.00$ & $0.46 \pm 0.01$ \\
APEP & $1.95 \pm 0.01$ & $2.60 \pm 0.02$ & $1.84 \pm 0.01$ & $2.13 \pm 0.01$ & $0.76 \pm 0.00$ & $1.16 \pm 0.01$ \\
\hline
\end{tabular}


a)

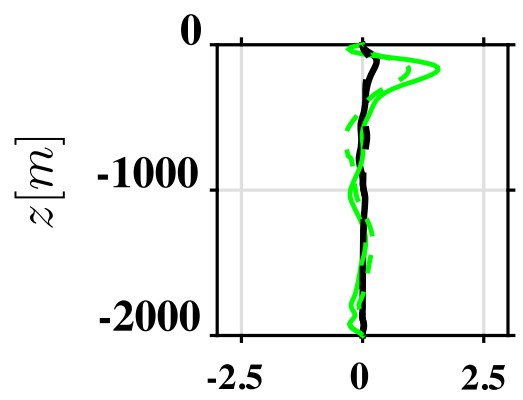

b)

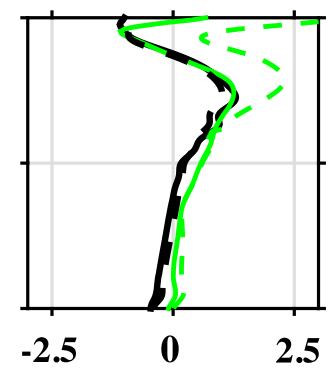

c)

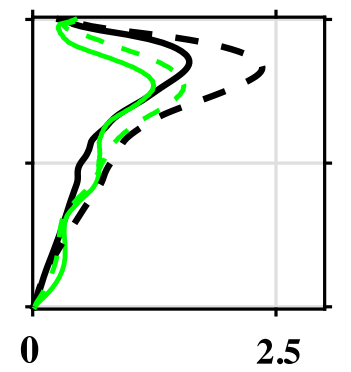

d)

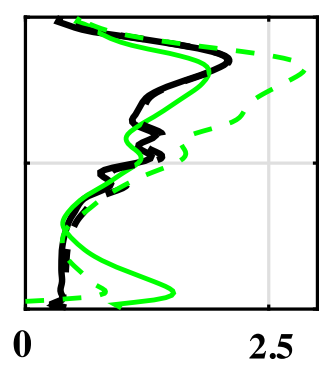

FIG. 9. Time- and horizontal-averaged vertical [(14)] and horizontal [(13)] components $\left(10^{-9} \mathrm{~W} \mathrm{~kg}^{-1}\right)$ of (a), (b) SP, (c) BP, and (d) APEP for the LFW (black) and COMB (green) solutions computed using $0.3 f$ (dashed) and $0.9 f$ (solid) Gaussian filter widths.

shear production, APE production, and buoyancy conversion using $\mathcal{G}_{0.9}$ (Fig. 10). In the LFW case, positive SP values (Fig. 10a) occur predominantly at the edges of the region of the large, anticyclonic eddy (see Figs. 1 and 3). Positive APEP values (Fig. 10c) are found deeper at the edges of the anticyclonic eddy and closer to the surface in the region between the anticyclone and the midsize cyclones. For the COMB case, signatures of waveinduced positive SP (Fig. 10d) are found in the interior of the anticyclonic eddy region as well as in the region of the midsize cyclonic eddies, extending deeper in both locations. Elevated values of APEP (Fig. 10f) are found in the anticyclonic eddy region close to the bottom. The BP structure is generally similar for the LFW and COMB solutions (Figs. 10b,e), with positive values extending deeper in the latter.

\section{Internal wave effects on coherent eddies}

We now focus on the effects the HF wind forcing and the excited IWs have on the large, coherent, anticyclonic eddy and midsize cyclonic eddies seen in Figs. 1, 3, and 4. These coherent eddies are not to be confused with the "eddies" discussed in section 4a, which were defined as a perturbation from a zonal mean.

The time- and zonally averaged EKE dissipation for the LFW and COMB solutions are shown in Fig. 11. There is a significant increase in dissipation in the COMB case both near the surface where submesoscale fronts and filaments are most active and at depth in the regions where the coherent eddies are found. This is in agreement with Fig. 4, the volume-averaged quantities in Fig. 7, and the spatial patterns of LF-HF energy conversion in Fig. 10. To compare the level of enhanced dissipation within the cyclonic and anticyclonic eddies we use the eddy tracking algorithm described in appendix C. There is a four/thirtyfold increase in the dissipation rate inside the anticyclonic/cyclonic eddies, respectively (Table 2). Accordingly, a representative pattern of the azimuthal- and radial-averaged dissipation within the cyclonic and anticyclonic eddies is much larger in the COMB solution (Fig. 11f). Inside the anticyclonic eddy, the largest dissipation increase is closer to the bottom, whereas inside the cyclonic eddies the dissipation increase is roughly constant with depth. These dissipation profiles imply that the interactions
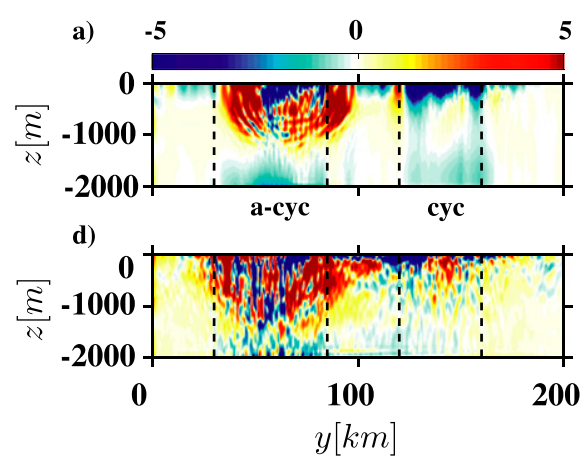

b) -2.5

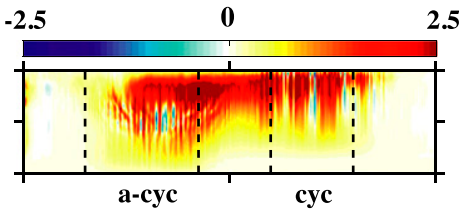

e)

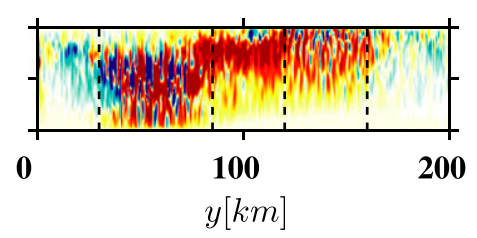

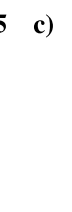

f)
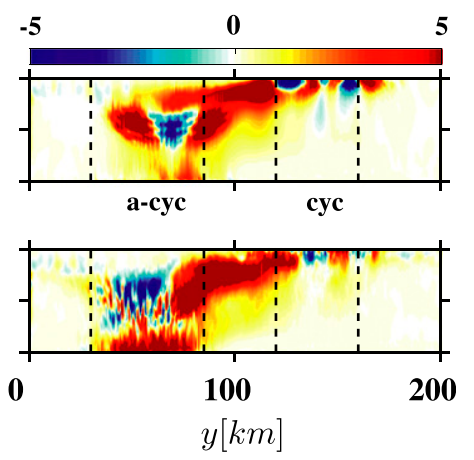

FIG. 10. (a),(d) Time- and zonal-averaged HF-LF SP [(10)], (b),(e) HF-HF BP [(9)], and (c),(f) HF-LF APEP [(11)] for the (top) LFW case and (bottom) COMB case $\left(10^{-9} \mathrm{~W} \mathrm{~kg}^{-1}\right)$. See (8) and appendix B for details. Dashed vertical lines denote the regions dominated by the anticyclonic eddy and midsize cyclonic eddies (see Fig. 3). 

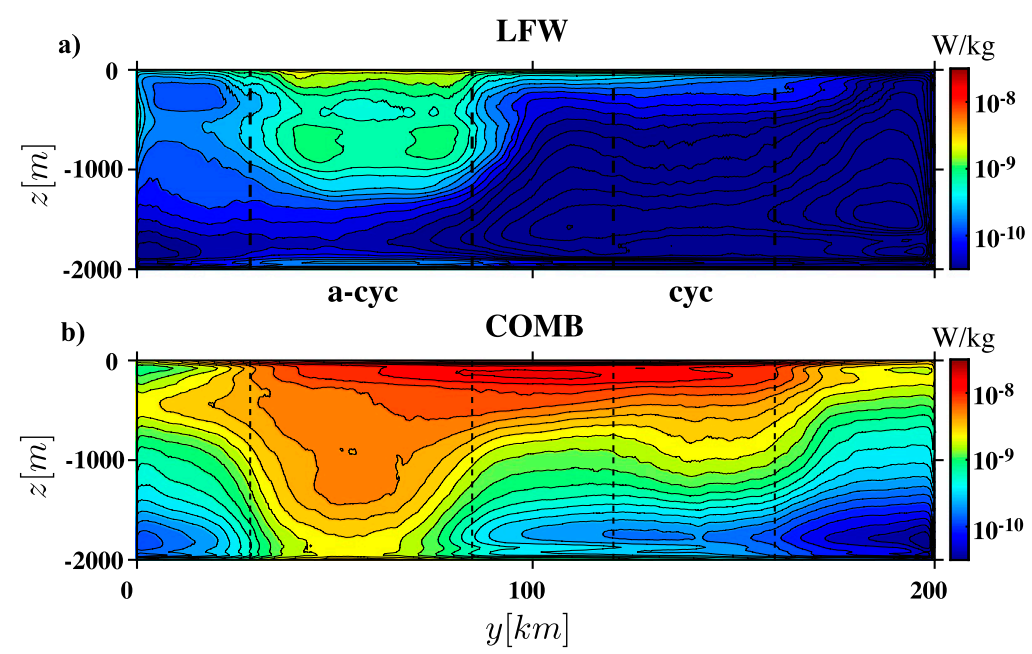
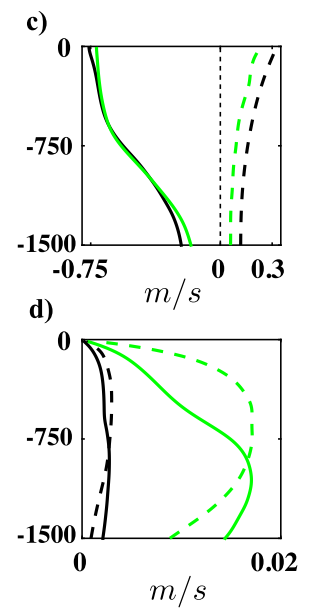

e)

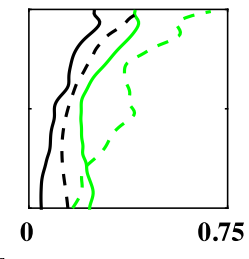

f)

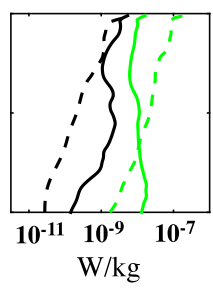

FIG. 11. Time- and zonal-averaged EKE dissipation for the (a) LFW solution and (b) COMB solution. Dashed vertical lines denote the regions dominated by the anticyclonic eddy and midsize cyclonic eddies (see Figs. 3,10). Azimuthal- and radial-averaged (c) azimuthal velocity, (d) RMS perturbation vertical velocity, (e) RMS perturbation Rossby number, and (f) log dissipation for representative snapshots of an anticyclonic eddy (solid lines) and cyclonic eddy (dashed lines) for the LFW (black lines) and COMB (green lines) solutions. Perturbations are from an azimuthal average.

with the externally forced IWs extend to depth and that they are manifested differently in cyclones and anticyclones. There is a corresponding increase in the rootmean-square (RMS) perturbation vertical velocity and Rossby number in the COMB solution for both cyclonic and anticyclonic eddies (Figs. 11d,e). The increase in RMS $w$ is largest between 300- and 1000-m depth for the cyclonic eddies and between 1000 and $1500 \mathrm{~m}$ for the anticyclonic eddy. The azimuthal velocity profile remains largely unchanged between LFW and COMB solutions in the anticyclonic eddy (Fig. 11c). Conversely, an approximately depth-uniform azimuthal velocity decrease is evident in the cyclonic eddy for the COMB solution.

Next, we compute the time- and eddy volumeaveraged energy conversion terms (9), (10), and (11) from mesoscale to IW frequencies (Table 3 ) in order to assess the significance of direct extraction in the coherent eddies. Despite the large standard errors, the following quantitative statements can be made: In the anticyclonic eddy total, horizontal, and vertical shear productions are positive in both the LFW and COMB solutions. There is an increase in the vertical shear production for the COMB solution; however, the large standard errors make it impossible to confirm that the reductions in the total and horizontal shear productions are statistically significant. BP in the COMB solution decreases to a negative value, indicating a conversion from HF $E_{k}$ to HF APE. The LF to HF APE transfer is marginally positive in both cases without any statistically significant change. There is thus little evidence in favor of direct extraction within the anticyclonic eddy.
Conversely, in the cyclonic eddies, total and horizontal shear productions are positive for the COMB solution, whereas the same quantities have large standard errors for the LFW solution indicating that the LF to HF $E_{k}$ transfer is often reversed. The vertical shear production is negative in both cases with no clear difference in magnitude. APE production is positive for both solutions and larger in COMB. This suggests that direct extraction is more prevalent within the cyclonic eddies.

Finally, we evaluate whether temporal energy transfers correspond to spatial energy transfers and compute the spatial conversion terms $\mathrm{SP}^{S}, \mathrm{APEP}^{S}$, and $\mathrm{BP}^{S}$ in the interior of the eddies (Table 4). The terms $\mathrm{SP}^{S}, \mathrm{APEP}^{S}$, and $\mathrm{BP}^{S}$ are similar to their temporal counterparts; however, instead of a temporal filter we apply a spatial two-dimensional symmetric Gaussian filter with filter widths of $7.8 \mathrm{~km}$. The filter widths were chosen to give low-pass spatial scales larger than $\sim 30 \mathrm{~km}$, in agreement with the large mesoscale structures shown in Figs. 1 and 3. Similarly to the temporal conversion terms, positive $\mathrm{SP}^{S}$ denotes $E_{k}$ transfer from large scales to small scales, positive $\mathrm{APEP}^{S}$ denotes large-scale to small-scale APE

TABLE 2. Time- and volume-averaged kinetic energy dissipation computed in the interior of the larger anticyclonic and cyclonic eddies and elsewhere in the top $1000 \mathrm{~m}$ (see appendix $\mathrm{C}$ for detail). All values are in $\mathrm{mW} \mathrm{m}^{-2}$.

\begin{tabular}{cccc}
\hline \hline Solution & Cyclonic & Anticyclonic & Elsewhere \\
\hline LFW & $1.4 \pm 0.0$ & $2.0 \pm 0.1$ & $0.3 \pm 0.0$ \\
COMB & $40.5 \pm 1.9$ & $8.3 \pm 0.5$ & $6.5 \pm 0.4$ \\
\hline
\end{tabular}


TABLE 3. Time- and volume-averaged temporal conversion terms $\left(\mathrm{mW} \mathrm{m}^{-2}\right)$ computed using $\mathcal{G}_{0.9}$ in the interior of the larger anticyclonic and cyclonic eddies over the top $850 \mathrm{~m}$ (see appendix $\mathrm{C}$ for detail). Bold values have large standard errors and are therefore not statistically significant.

\begin{tabular}{cccrrr}
\hline \hline & \multicolumn{4}{c}{ Cyclonic } \\
\cline { 2 - 6 } Conversion term & SP & \multicolumn{1}{c}{ SP $_{\text {hor }}$} & SP $_{\text {ver }}$ & BP & APEP \\
\hline LFW & $\mathbf{7 . 4} \pm \mathbf{8 . 4}$ & $\mathbf{8 . 5} \pm \mathbf{8 . 4}$ & $-1.1 \pm 0.1$ & $2.8 \pm 0.8$ & $4.4 \pm 1.9$ \\
COMB & $7.5 \pm 4.7$ & $8.9 \pm 4.5$ & $-1.4 \pm 0.4$ & $\mathbf{0 . 4} \pm \mathbf{1 . 1}$ & $8.4 \pm 1.0$ \\
& & & Anticyclonic & & \\
LFW & $47.9 \pm 16.9$ & $46.4 \pm 16.8$ & $1.5 \pm 0.2$ & $\mathbf{0 . 0} \pm \mathbf{0 . 3}$ & $1.7 \pm 1.3$ \\
COMB & $26.0 \pm 10.6$ & $21.7 \pm 10.5$ & $4.3 \pm 0.5$ & $-2.4 \pm 0.5$ & $\mathbf{1 . 4} \pm \mathbf{1 . 3}$ \\
\hline
\end{tabular}

transfer, and positive $\mathrm{BP}^{S}$ denotes an energy conversion from small-scale APE to small-scale $E_{k}$.

In the anticyclonic eddy, there is an increase in $\mathrm{SP}^{S}$ and $\mathrm{SP}_{\text {hor }}^{S}$ from negative values in the LFW solution to marginally positive values in the COMB solution and a smaller decrease in $\mathrm{SP}_{\mathrm{ver}}^{S}$. In the cyclonic eddies, $\mathrm{SP}^{S}$, $\mathrm{SP}_{\text {hor }}^{S}$, and $\mathrm{SP}_{\mathrm{ver}}^{S}$ in the COMB solution all increase and are positive, indicating a transfer from large-scale to small-scale $E_{k}$. Similarly, $\mathrm{APEP}^{S}$ in the COMB solution increases and is positive, indicating a transfer from large-scale to small-scale APE. These findings demonstrate that the forward LF to HF energy transfer in the cyclonic eddies correspond to a forward large-scale to small-scale transfer.

In both the anticyclone and the cyclones $\mathrm{BP}^{S}$ of the COMB solution is reduced, implying less small-scale APE is converted to small-scale $E_{k}$. The large standard errors indicate a continuous back and forth exchange of small-scale kinetic and small-scale available potential energies.

\section{Summary and discussion}

Submesoscale-resolving numerical simulations of an idealized ACC are used to investigate the interaction between energetic mesoscale and submesoscale flows, generated by steady [low frequency (LF)] wind forcing and an internal wave (IW) field, excited by stochastic [high frequency $(\mathrm{HF})]$ wind forcing. In particular, we aim to distinguish between stimulated imbalance, a mechanism through which externally forced IWs trigger a forward mesoscale to submesoscale eddy kinetic energy (EKE) cascade to dissipation, and direct extraction mechanisms of mesoscale EKE by IWs, followed by a forward IW energy cascade to dissipation. To this end we examine three solutions: one forced by HF wind only (HFW), one forced by LF wind only (LFW), and one by a combination of the two (COMB).

The HFW solution is characterized by an active IW field predominately at near-inertial frequencies. The LFW solution is characterized by a larger anticyclonic eddy, two midsize cyclonic eddies along with smaller fronts, filaments, and eddies, all of which have Ro $O(1)$. The COMB solution combines the characteristic phenomena in the HFW and LFW solutions, resulting in a more active submesoscale field, with smaller spatial and temporal scales and more abundant fronts and filaments (Figs. 1, 3-6).

Although neither the LFW nor the HFW frequency spectral slopes independently match observations at either sub- or superinertial frequencies, the COMB solution does (Fig. 5). This suggests that the interactions among IWs mesoscales and submesoscales are significant at a wide range of temporal and spatial scales, as is manifested by the resulting shallower frequency and wavenumber spectra. In addition, it implies that more energy is being transferred from LF and large-scale motions to higher frequencies and smaller scales.

TABLE 4. Time- and volume-averaged spatial conversion terms $\left(\mathrm{mW} \mathrm{m}^{-2}\right)$ computed in the interior of the larger anticyclonic and cyclonic eddies over the top $850 \mathrm{~m}$ (see appendixes B and C for detail). A horizontally symmetric Gaussian filter was used with a $7.8 \mathrm{~km} \times$ $7.8 \mathrm{~km}$ filter area. Bold values have large standard errors and are therefore not statistically significant.

\begin{tabular}{crccrr}
\hline \hline & \multicolumn{5}{c}{ Cyclonic } \\
\cline { 2 - 6 } Conversion term & \multicolumn{1}{c}{$\mathrm{SP}^{S}$} & $\mathrm{SP}_{\text {hor }}^{S}$ & \multicolumn{1}{c}{$\mathrm{SP}_{\text {ver }}^{S}$} & \multicolumn{1}{c}{$\mathrm{BP}^{S}$} & \multicolumn{1}{c}{$\mathrm{APEP}^{S}$} \\
\hline LFW & $\mathbf{- 0 . 1} \pm \mathbf{0 . 6}$ & $1.2 \pm 0.6$ & $-1.3 \pm 0.1$ & $154.6 \pm 65.0$ & $972.0 \pm 720.5$ \\
COMB & $10.7 \pm 2.7$ & $6.4 \pm 2.5$ & $4.3 \pm 1.4$ & $-\mathbf{1 2 8 . 4} \pm \mathbf{1 8 5 . 3}$ & $1861.5 \pm 295.9$ \\
& & & Anticyclonic & & $-\mathbf{9 0 . 8} \pm \mathbf{3 4 3 . 4}$ \\
LFW & $-20.5 \pm 2.5$ & $-24.5 \pm 2.5$ & $4.0 \pm 0.1$ & $736.9 \pm 71.7$ & $\mathbf{1 7 . 4} \pm \mathbf{2 5 2 . 6}$ \\
COMB & $\mathbf{1 . 8} \pm \mathbf{3 . 5}$ & $-\mathbf{0 . 6} \pm \mathbf{3 . 5}$ & $2.3 \pm 1.0$ & $-\mathbf{8 6 . 4} \pm \mathbf{3 9 8 . 7}$ & \\
\hline
\end{tabular}


The fact that the COMB frequency and wavenumber spectral slopes match the GM ones, whereas the HFW spectral slopes do not, suggests that mesoscale and submesoscale motions can affect the HF energy distribution. Previous studies (Van Meurs 1998; Ponte and Klein 2015) have discussed the possibility for the scattering of IWs by mesoscale features; however, they did not consider the full nonlinear interactions between the scattered IWs and the balanced motions. More work is required to establish how much of the HF small-scale energy content is associated with IWs versus other unbalanced motions.

\section{a. Stimulated imbalance versus direct extraction mechanisms}

To establish that the IW-mediated routes to dissipation are important in our solutions we compute $R_{\mathrm{IW}_{\epsilon}}$, the ratio between the enhanced dissipation to the added HF wind work, namely,

$$
R_{\mathrm{IW}_{\epsilon}}=\frac{\epsilon_{\mathrm{COMB}}-\epsilon_{\mathrm{LFW}}}{\tau_{\text {work }_{\mathrm{COMB}}}-\tau_{\text {work }_{\mathrm{LFW}}}}=1.3 .
$$

The fact that $R_{\mathrm{IW}_{\epsilon}}>1$ implies that a fraction of the energy that is dissipated must be transferred from LF motions. In the COMB solution this amounts to $0.9 \mathrm{~mW} \mathrm{~m}^{-2}$ (Fig. 7), a quantity we refer to as excess dissipation in section $4 \mathrm{a}$.

One can estimate the importance of the forward EKE cascade in these solutions, either by stimulated imbalance or direct extraction mechanisms, by comparing the ratio between the small-scale EKE dissipation $\epsilon^{\prime}$ to the total EKE dissipation $\epsilon^{\prime}+\epsilon_{d}^{\prime}$ (Fig. 7). For the LFW solution this ratio is $\approx 0.14$, and for the COMB solution it is $\approx 0.37$, where for the latter we have used the excess dissipation instead of the total small-scale EKE dissipation to only take into account the amount of energy that is necessarily transferred from LF motions. This shows that in our solutions externally forced IWs can substantially increase the forward energy cascade to dissipation. Furthermore, we can define the dissipation enhancement efficiency as $R_{\mathrm{IW}_{\epsilon}}-1=0.3$. Observations indicate that the wind work on NIWs is $0.5-1.1 \mathrm{TW}$ (Alford 2003; Jiang et al. 2005; Rimac et al. 2013), and consequently the 0.3 efficiency suggests that IWs could potentially lead to the transfer of $0.15-0.33 \mathrm{TW}$ from the balanced geostrophic EKE reservoir. Note, however, that the dynamical regime of our simulations are representative of the more energetic parts of the ocean, such as the ACC and the western boundary currents, where the Ro number is frequently $O(1)$ even at mesoscales. In more quiescent regions, where the Ro is smaller, the dissipation enhancement efficiency may be lower. More work is required to establish the sensitivity of these interactions to the Ro number magnitude.

To assess whether the IW-induced geostrophic EKE transfer to dissipation is a result of direct extraction mechanisms or stimulated imbalance we apply a filtering approach (section $4 \mathrm{~b}$ ) to analyze the LF to HF $E_{k}$ and APE transfers. Using filter widths of $48\left(\mathcal{G}_{0.3}\right)$ and $16 \mathrm{~h}$ $\left(\mathcal{G}_{0.9}\right)$, we are able to distinguish between three frequency bands: low, intermediate, and high, which we associate with mesoscale, submesoscales, and IWs, respectively (Fig. 8). We attribute energy transfers from mesoscale to submesoscale frequency bands to stimulated imbalance and energy transfers from mesoscales to IW frequency bands to direct extraction. This approach was tested with an additional filter width (section $4 \mathrm{~b}$ and Table 1) to show that despite the frequency overlap between the different motions and the limitation of the filtering approach, the distinction between direct extraction and stimulated imbalance is robust. Figure 12 illustrates schematically the two possible IW-mediated mesoscale energy routes to dissipation as well as the independent dissipation route associated with the added HF wind work.

We find an enhanced LF to HF transfer of $E_{k}$ in the COMB solution, where about half of the LF $E_{k}$ is transferred to submesoscale frequencies, predominantly due to $\mathrm{SP}_{\text {hor }}[(13)]$, and another half is transferred all the way to IW frequencies, predominantly due to $\mathrm{SP}_{\mathrm{ver}}$ [(14); Table 1 and Figs. 9 and 10]. This implies that both direct extraction mechanisms and stimulated imbalance are important in this case. Taylor and Straub (2016) have also observed an induced LF to HF $E_{k}$ transfer in the presence of IWs. Their mesoscalepermitting resolution, however, suggests that stimulated imbalance could not have been resolved in their solutions. The reduction in BP [(9)] for the COMB solution implies that less HF APE is converted to HF $E_{k}$, and the filter width comparison suggests the reduction occurs at submesoscale frequencies. We associate this reduction with the, apparently, more efficient forward route for submesoscale APE provided by stimulated imbalance and direct extraction mechanisms, as indicated by the increase in LF to HF transfer of APE (APEP) in the COMB solution. This increase in APEP is to submesoscale frequencies in the upper ocean and to IW frequencies at depth. It illustrates another pathway for $E_{k}$ dissipation whereby LF APE is converted to HF APE, which, in turn, is converted to $\mathrm{HF} E_{k}$ via $\mathrm{BP}$ and is eventually dissipated. Note that a careful quantitative analysis of LF-HF APE transfers for high Ro flows requires a more general definition of APE (Winters et al. 1995; Winters and Barkan 2013) and is beyond the scope of this paper. 


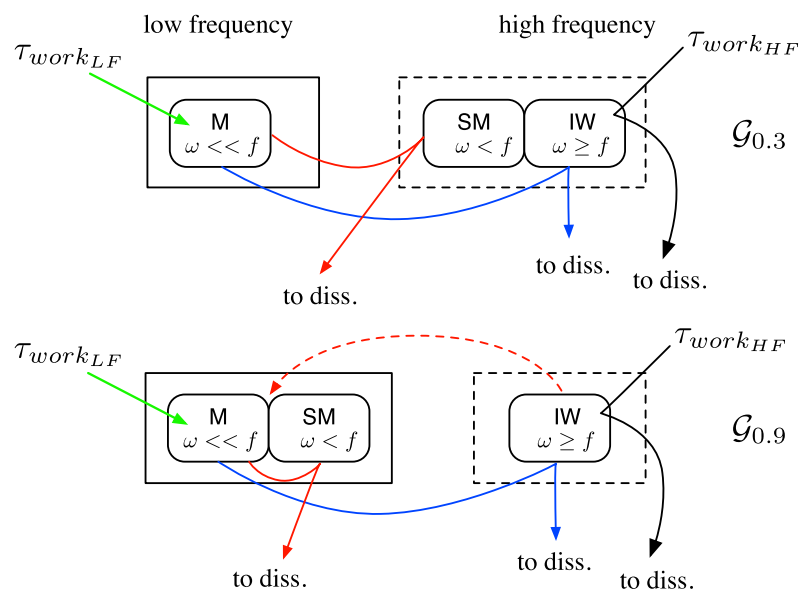

FIG. 12. A schematic illustrating the LF to HF energy pathways in the COMB solution associated with the $\mathcal{G}_{0.3}$ and $\mathcal{G}_{0.9}$ filter widths. The solid green arrow represents the generation of mesoscale (M) energy by LF wind work $\tau_{\text {work }}$. The solid black arrow represents the dissipation route associated with the added $\mathrm{HF}$ wind work $\tau_{\text {work }_{\mathrm{HF}}}$. The solid blue arrow represents the dissipation route associated with direct extraction mechanisms, such as stimulated emission. The solid red arrow represents the dissipation route associated with stimulated imbalance. The dashed red line illustrates that the forward $\mathrm{M}$ to submesoscale (SM) energy cascade is stimulated by the IW field. See text for detail.

\section{b. Comparison with existing theories}

In the asymptotic theory of XV15, two conservation laws are derived for the NIW-QG coupled system. The first is for the total (kinetic + potential) QG energy and NIW (available) potential energy. The second is for the wave action, which for NIWs, implies conservation of NIW kinetic energy. ${ }^{2}$ Thus, according to XV15, any increase in wave APE is directly extracted from total QG energy, a mechanism they refer to as stimulated emission. Stimulated emission is an example of a direct extraction mechanism that is presumed to lead to a forward IW energy cascade to dissipation. Note that because stimulated emission relies on energy transfer to IW APE, it must be associated with LF to HF APE transfer. XV15 provide the following scaling for the excess dissipation $\epsilon_{\mathrm{exc}_{\mathrm{XV} 15}}$ due to energy exchanges between NIWs and geostrophic flows:

$\epsilon_{\mathrm{exc}_{\mathrm{XV} 15}}=\left(\tau_{\text {work }_{\mathrm{COMB}}}-\tau_{\text {work }_{\mathrm{LFW}}}\right) \times \frac{1}{2}\left(\frac{N k_{h}}{f m}\right)^{2}$,

where $N^{2} k_{h}^{2} f^{-2} m^{-2}$ is the wave Burger number $\mathrm{Bu}$, and $k_{h}(m)$ are the horizontal (vertical) wavenumbers.

\footnotetext{
${ }^{2}$ The theory of XV15 does not account for Doppler shifting of the IW frequency.
}

Following Young and Ben-Jelloul (1997) and XV15, we scale $^{3}$ the geostrophic velocity using $U_{g} \sim \mathrm{Buf} / k_{h}$ and consequently the geostrophic Rossby number $\mathrm{Ro}_{g} \sim$ $U_{g} k_{h} / f \sim \mathrm{Bu}$.

We can thus estimate an upper bound on the amount of excess dissipation due to stimulated emission by estimating $\operatorname{Ro}_{g}$ locally in our solutions as $\tilde{\zeta}_{\text {nd }} / f$, where $\tilde{\zeta}_{\text {nd }}$ is the vertical component of vorticity associated with the low-pass filtered (using $\mathcal{G}_{0.9}$ ) and horizontally nondivergent (computed using a Helmholtz decomposition) part of the velocity field. The upper bound on the time-

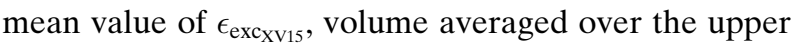
$100 \mathrm{~m}$ in the COMB solution is $0.33 \mathrm{~mW} \mathrm{~m}^{-2}$.

WT15 proposed a direct extraction mechanism that relies on LF to HF kinetic energy transfer associated with the lateral shear production exerted by near inertial oscillations on a geostrophic current. They propose the following scaling for the excess dissipation $\epsilon_{\mathrm{exc}_{\mathrm{WT} 15}}$ :

$\epsilon_{\mathrm{exc}_{\mathrm{WT} 15}}=\left(\tau_{\text {work }_{\mathrm{COMB}}}-\tau_{\text {work }_{\mathrm{LFW}}}\right) \times\left[\frac{\mathrm{Ro}_{g}^{2}}{4\left(1+\mathrm{Ro}_{g}\right)}\right]$.

The time- and volume-averaged $\epsilon_{\mathrm{exc} \mathrm{WT}_{15}}$ over the upper $100 \mathrm{~m}$ in the COMB solution is $0.14 \mathrm{~mW} \mathrm{~m}^{-2}$. Therefore, the combined excess dissipation due to the above direct extraction mechanisms is, at most, half of the observed $0.9 \mathrm{~mW} \mathrm{~m}^{-2}$ computed in our solutions. Note that these theories focus on IW generation, whereas we demonstrate that energy exchanges are prevalent in regions that extend deep in the water column and are linked to the coherent cyclonic and anticyclonic eddies (Fig. 10). Indeed, dissipation rates, RMS Ro, and RMS $w$ are all substantially amplified in the eddy interiors in the presence of IWs (Fig. 11).

\section{c. The interaction of IWs with coherent cyclones and anticyclones}

To investigate the interactions between IWs and the larger coherent anticyclone and cyclones, we computed the time- and volume-averaged temporal (SP, BP, and $\mathrm{APEP})$ and spatial $\left(\mathrm{SP}^{S}, \mathrm{BP}^{S}, \mathrm{APEP}^{S}\right)$ energy conversion terms in the interior of the eddies (Tables 3,4). This allowed us to determine whether there is a connection between forward energy transfers in temporal and spatial scales and assess the differences between cyclones and anticyclones. The temporal conversion terms were computed using the $\mathcal{G}_{0.9}$ to focus on transfers to IW frequencies (direct extraction).

\footnotetext{
${ }^{3}$ This comes from their scaling choice to obtain a "leadingorder" IW-QG interaction.
} 
Inside the cyclonic eddies, $E_{k}$ and APE are transferred to IW frequencies and smaller scales in the COMB solution. The large standard errors associated with this computation (appendix C) make it difficult to quantify which of the energy transfers, spatial or temporal, is amplified more compared with the LFW solution. Nevertheless, this analysis suggests that there is a connection between the two and that direct extraction may be important.

Inside the anticyclonic eddy LF $E_{k}$ and APE are transferred to HF $E_{k}$ and APE in the IW frequency band for both LFW and COMB solutions, without any statistically significant enhancement in the latter. Spatially, there is an inverse horizontal $E_{k}$ transfer to large scales in the LFW solution that is arrested in the COMB solution. This implies that direct extraction is less prevalent in this case and illustrates the difference between IW-cyclone and IW-anticyclone interactions.

\section{d. Final remarks}

We hypothesize that stimulated imbalance may be associated with IW-triggered instabilities. Further understanding of the nature of submesoscale instabilities of high Ro eddies, fronts, and filaments is required to understand the way in which IWs can stimulate them, thereby leading to a forward energy cascade. Another possibility is that stimulated imbalance is associated with LF to IW extraction followed by an IW to submesoscale transfer, as suggested by Thomas (2012).

In our solution analysis it was presumed that time scales of $O(2-4)$ days are associated with submesoscale motions and that time scales shorter than a day are associated with IWs; however, Doppler shifting of IWs by lower-frequency motions is also a possibility. Removing the effects of Doppler shifting by applying Lagrangian frame temporal filters, generalizing the methodology described in Nagai et al. (2015), was not attempted in this study and is left to future work. Note that an accurate quantification of IW-submesoscale energy exchanges relies on having a clear scale separation between the two motions, even in a Lagrangian reference frame, but submesoscale advective features of $O(f)$ and higher are not entirely implausible. Furthermore, it is not obvious how linear are the IWs in these solutions and therefore whether they are expected to obey the classical IW dispersion and polarization relations. These outstanding issues have strong implications for any theory of wave-vortex interaction.

Acknowledgments. This work was supported by the National Science Foundation (OCE-1061027 and ONR N00014141062). XSEDE computing resources at the National Institute of Computational Sciences, University of Tennessee, were made available under Grants TGOCE120004 and TG-OCE150010. We thank the editor and two anonymous reviewers for helpful suggestions to improve the presentation and interpretation of our results.

\section{APPENDIX A}

\section{Details of Numerical Simulations}

The Boussinesq equations of motion solved by the model are

$$
\begin{aligned}
\frac{\partial u_{i}}{\partial t}+u_{k} \frac{\partial u_{i}}{\partial x_{k}}+f \epsilon_{3 m i} u_{m}= & -\frac{\partial p}{\partial x_{i}}+b \delta_{i 3}+D\left(u_{i}\right) \\
& -r F_{d}\left(x_{3}\right) u_{j} \delta_{i j}+F_{\tau}\left(x_{3}\right) \\
\frac{\partial b}{\partial t}+u_{k} \frac{\partial b}{\partial x_{k}} & =D(b), \text { and } \\
\frac{\partial u_{i}}{\partial x_{i}} & =0,
\end{aligned}
$$$$
-r F_{d}\left(x_{3}\right) u_{j} \delta_{i j}+F_{\tau}\left(x_{3}\right) \delta_{i 1}
$$

where the density is expressed as $\rho_{0}\left(1-g^{-1} b\right), b$ is the buoyancy, $\rho_{0} p$ is the pressure, $\mathbf{u}=u_{i}=(u, v, w), x_{i}=$ $(x, y, z), f$ is the Coriolis frequency, $r$ is bottom drag, $\epsilon_{l m i}$ is the Levi-Civita symbol, $\delta$ is the Kronecker delta, $i, k=$ $1-3, j=1,2$, and summation over repeated indices is assumed. The diffusive terms

$$
\begin{aligned}
& D\left(u_{i}\right)=\nu \frac{\partial^{2} u_{i}}{\partial x_{k} \partial x_{k}}+\nu_{h}^{*} \frac{\partial^{8} u_{i}}{\partial x_{j}^{8}}+\nu_{v}^{*} \frac{\partial^{4} u_{i}}{\partial x_{3}^{4}}, \quad \text { and } \\
& D(b)=\kappa \frac{\partial^{2} b}{\partial x_{k} \partial x_{k}}+\kappa_{h}^{*} \frac{\partial^{8} b}{\partial x_{j}^{8}}+\kappa_{v}^{*} \frac{\partial^{4} u_{i}}{\partial x_{3}^{4}},
\end{aligned}
$$

where $\nu=50 \kappa=5 \times 10^{-4} \mathrm{~m}^{2} \mathrm{~s}^{-1}$, and $\nu_{h}^{*}, \kappa_{h}^{*}$ and $\nu_{v}^{*}, \kappa_{v}^{*}$ are the horizontal and vertical hyperdiffusive terms, respectively. See Ulloa et al. (2015) and Winters (2015) for a detailed discussion of hyperviscosity in flow_solve. The form of the bottom $\operatorname{drag} F_{d}(z)$ in (A.1a) is

$$
F_{d}(z)=\frac{H}{\sigma_{d}} \sqrt{\frac{2}{\pi}} e^{-\left[(z+H) /\left(\sqrt{2} \sigma_{d}\right)\right]^{2}},
$$

where $\sigma_{d}=2 d z$. Equation (A.6) is designed to have a depth-averaged value of 1 .

The surface stresses (1), (2), and (3) are applied as a body force $F_{\tau}(z)$ in the zonal momentum equation [(A.1a)], which takes the form

$$
F_{\tau}(z)=\frac{1}{\delta_{\mathrm{SBL}}} \sqrt{\frac{2}{\pi}} e^{-\left[z /\left(\sqrt{2} \delta_{\mathrm{SBL}}\right)\right]^{2}} \tau_{(s, w, \text { total })},
$$


where $\delta_{\mathrm{SBL}}=5 d z$ is chosen to distribute the stressinduced momentum over a resolvable thin layer near the top of thickness $O(5 d z)$, effectively constraining the thickness of the momentum SBL to be of the order of the turbulent Ekman layer depth $\sim u^{*} / f \approx 80 \mathrm{~m}$, where $u^{*}=\sqrt{\tau_{s} / f}$ is the frictional velocity. This is in general agreement with the more traditional KPP (Large et al. 1994) boundary layer parameterization, where if only wind forcing is applied the SBL depth in unstratified regions will be the turbulent Ekman layer depth. Although this body force implementation augmented by the hyperviscous operators is inferior to KPP, we argue it should not too greatly affect the results. Figure 2 shows that the layer of mixed fluid in the anticyclonic flank of the jet can reach a depth of approximately $200 \mathrm{~m}$. This demonstrates that the SBL depth in these solutions is still largely affected by the flow field and therefore spatially and temporally varying. Similarly, Figs. 4 and $11 \mathrm{dem}-$ onstrate that the near-surface energy dissipation (and momentum mixing) is varying as well. We have tested a LFW solution where we doubled the vertical resolution and have not noticed any clear quantitative or qualitative difference. We therefore reverted to the current vertical resolution to economize on computing resources

The volume-averaged wind work in (4) is defined as

$$
\tau_{\text {work }}=\left\langle u F_{\tau}\right\rangle \text {. }
$$

Similarly, the volume-averaged bottom drag dissipation is defined as

$$
r\left\langle u_{i} \mathcal{F}_{d} x_{3} u_{j} \delta_{i j}\right\rangle
$$

Finally, the difference between the kinetic energy dissipation computed as in (5) versus multiplying (A.2a) by $u_{i}$ and volume averaging is of order of the machine precision. The reader is referred to Barkan et al. $(2013,2015)$ for a more detailed demonstration of the ability of flow_solve to accurately conserve kinetic and available potential energies.

The output files consist of three-dimensional velocity, buoyancy, and pressure fields saved once every $12 \mathrm{~h}$ and two-dimensional velocity, buoyancy, and pressure cross sections at selected locations saved once every hour. In addition, during 1 month of the simulation after equilibrium has been reached the full three-dimensional fields are saved once every hour.

\section{APPENDIX B}

\section{Derivation of the Filtered Energy Equations}

We define a filter $\mathcal{G}(t)$ acting on any function $\phi(\mathbf{x}, t)$ such that the filtered field (denoted by tilde) is

$$
\overline{\phi(\mathbf{x}, t)}=\int \mathcal{G}(t-\hat{t}) \phi(\mathbf{x}, \hat{t}) d \hat{t} .
$$

Applying the filtering operation (B.1) to (A.1a) and (A.1c), the inviscid filtered momentum equations become

$$
\frac{\partial \widetilde{u}_{i}}{\partial t}+\frac{\partial}{\partial x_{k}} \widetilde{u_{i} u_{k}}+f \epsilon_{3 m i} \widetilde{u_{m}}=-\frac{\partial \tilde{p}}{\partial x_{i}}+\tilde{b} \delta_{i 3},
$$

and we further assume that

$$
\frac{\partial \widetilde{u}_{i}}{\partial x_{i}}=0 .
$$

Following Leonard (1974), we split

$$
\widetilde{u_{i} u_{k}}=\tilde{u}_{i} \tilde{u}_{k}+\tau_{i k}^{r}
$$

and (B.2) becomes

$\frac{\partial \widetilde{u}_{i}}{\partial t}+\widetilde{u}_{k} \frac{\partial}{\partial x_{k}} \widetilde{u}_{i}+f \epsilon_{3 m i} \widetilde{u_{m}}=-\frac{\partial \tilde{p}}{\partial x_{i}}+\tilde{b} \delta_{i 3}-\frac{\partial \tau_{i k}^{r}}{\partial x_{k}}$.

Next we derive the filtered kinetic energy equation by multiplying (B.4) with $\tilde{u}_{i}$ to get

$\frac{D}{D t}\left(\frac{1}{2} \widetilde{u}_{i} \tilde{u}_{i}\right)+\frac{\partial}{\partial x_{k}}\left(\tilde{u}_{i} \tau_{i k}^{r}+\tilde{u}_{i} \tilde{p} \delta_{i k}\right)=\tilde{w} \tilde{b}+\tau_{i k}^{r} \frac{\partial \tilde{u}_{i}}{\partial x_{k}}$,

where $D / D t=\partial / \partial t+\tilde{u}_{k} \partial / \partial x_{k}$.

Finally, we derive the filtered kinetic energy equation for the perturbation by subtracting (B.5) from the filtered total kinetic energy equation

$$
\frac{D}{D t}\left(\frac{1}{2} \widetilde{u_{i} u_{i}}\right)+\frac{\partial}{\partial x_{k}}\left(E_{k}^{r}+\widetilde{u_{i} p} \delta_{i k}\right)=\widetilde{w b}
$$

to obtain

$$
\frac{D}{D t} E_{k_{\mathrm{HF}}}+\frac{\partial}{\partial x_{k}}\left(\mathrm{LSW}_{k}+\mathrm{PW}_{k}\right)=\mathrm{BP}+\mathrm{SP},
$$

where $E_{k_{\mathrm{HF}}}$ is defined in (8a), the Leonard stress work term

$$
\begin{aligned}
\mathrm{LSW}_{k} & \equiv E_{i k}^{r}-\tilde{u}_{i} \tau_{i k}^{r}, \quad \text { and } \\
E_{k}^{r} & \equiv u_{k} \frac{1}{2} u_{i} u_{i}-\tilde{u}_{k} \frac{1}{2} \widetilde{u_{i} u_{i}},
\end{aligned}
$$

the pressure work term

$$
\mathrm{PW}_{k} \equiv\left(\widetilde{u_{i} p}-\tilde{u}_{i} \tilde{p}\right) \delta_{i k},
$$

and the buoyancy conversion and shear production terms are defined in (9) and (10), respectively. 
Similarly, using (A.1b) and repeating similar steps, the evolution equation for the high-frequency buoyancy variance is

$$
\frac{D}{D t} \mathrm{BV}_{\mathrm{HF}}+\frac{\partial}{\partial x_{k}}\left(\mathrm{BW}_{k}\right)=\mathrm{BVP},
$$

where $\mathrm{BV}_{\mathrm{HF}}=N^{2} \mathrm{APE}_{\mathrm{HF}}$,

$$
\begin{aligned}
\mathrm{BW}_{k} & \equiv\left(\widetilde{u_{k} \frac{b^{2}}{2}}-\tilde{u}_{k} \frac{\tilde{b^{2}}}{2}\right)-\tilde{b} B_{k}, \quad \text { and } \\
\mathrm{BVP} & \equiv-B_{k} \frac{\partial \tilde{b}}{\partial x_{k}},
\end{aligned}
$$

and $B_{k}$ is defined in (12). Dividing (B.11) by $N^{2}$ and integrating by parts $z$ derivatives we arrive at $(8 b)$, where

$$
\begin{aligned}
\operatorname{APEW}_{k} & =\left(\widetilde{u_{k} \frac{b^{2}}{2 N^{2}}}-\tilde{u}_{k} \frac{\widetilde{b^{2}}}{2 N^{2}}\right), \quad \text { and } \\
\mathrm{BVW}_{3} & =\left(\widetilde{w \frac{b^{2}}{2}}-\tilde{w} \frac{b^{2}}{2}\right) .
\end{aligned}
$$

It was further assumed that $\partial \tilde{b} / \partial z \approx N^{2}$, which is certainly true for the time-averaged quantities presented in this paper. The Gaussian filters used in this study take the form

$$
\mathcal{G}(t)=\Delta t \frac{1}{\sqrt{2 \pi} \sigma} e^{-(t-\bar{t})^{2} / 2 \sigma^{2}}
$$

where $\Delta t$ is the time between model outputs, $\bar{t}$ is the mean of the output time series, $\sigma$ is the standard deviation, and the filter width is $2 \sigma$. For instance, the $\mathcal{G}_{0.3}$ filter has a standard deviation $\sigma=24 \mathrm{~h}$.

In section 5 and Table 3 , we compute $\mathrm{SP}^{S}, \mathrm{APEP}^{S}$, and $\mathrm{BP}^{S}$, which are identical to (10), (11), and (9), respectively, for spatial as opposed to temporal filters.

\section{APPENDIX C}

\section{Eddy Tracking Algorithm}

To compute volume-averaged quantities in the interior of the large anticyclonic eddy and the midsize cyclonic eddies, we used the following eddy tracking algorithm. For each snapshot, we applied at the surface a symmetric, two-dimensional Gaussian filter with filter area of $7.8 \mathrm{~km} \times 7.8 \mathrm{~km}$. Cyclonic eddies were defined as the regions with low-pass filtered Ro $>0.35$ in the northern half of the domain $(y>100 \mathrm{~km})$, and the anticyclonic eddy was defined as the region with low-pass filtered Ro $<-0.35$ in the southern half of the domain $(y<100 \mathrm{~km})$. This worked well because throughout the simulations the large anticyclonic/cyclonic eddies were indeed confined to the southern/northern half of the domain, respectively. The threshold Ro was chosen such that the fraction of the domain in which an eddy is wrongly identified (i.e., the fraction of the domain outside of the large anticyclonic eddy and midsize cyclonic eddies that satisfies the threshold criterion) is less than $0.01 \%$.

The eddy volume-averaged dissipation in Table 2 is computed using the above algorithm over the top half of the domain $(z>-1000 \mathrm{~m})$ once per day and then time averaged. The eddy volume-averaged energy conversion terms shown in Tables 3 and 4 are computed using the above algorithm over the top $850 \mathrm{~m}$ once per day and then time averaged. We picked a smaller domain for the energy conversion terms in order to reduce standard errors. The large computational cost and disk space requirements of computing three-dimensional conversion fields did not allow us to do it more frequently and resulted in considerable standard errors (bold numbers in Tables 3 and 4 indicate where the standard errors are too large and computed values are not statistically significant). The differences between the identified cyclonic and anticyclonic volumes in the LFW and COMB solutions were less than $5 \%$, indicating that a comparison between them is robust.

\section{REFERENCES}

Alford, M. H., 2003: Improved global maps and 54-year history of wind-work on ocean inertial motions. Geophys. Res. Lett., 30, 1424, doi:10.1029/2002GL016614.

Barkan, R., K. B. Winters, and S. G. L. Smith, 2013: Rotating horizontal convection. J. Fluid Mech., 723, 556-586, doi:10.1017/ jfm.2013.136.

,$- \ldots$, and _ 2015: Energy cascades and loss of balance in a reentrant channel forced by wind stress and buoyancy fluxes. J. Phys. Oceanogr., 45, 272-293, doi:10.1175/JPO-D-14-0068.1.

Bühler, O., and M. E. McIntyre, 2003: Remote recoil: A new wavemean interaction effect. J. Fluid Mech., 492, 207-230, doi:10.1017/S0022112003005639.

$\longrightarrow$, and —, 2005: Wave capture and wave-vortex duality. J. Fluid Mech., 534, 67-95, doi:10.1017/S0022112005004374.

Capet, X., J. C. McWilliams, M. J. Molemaker, and A. F. Shchepetkin, 2008: Mesoscale to submesoscale transition in the California Current System. Part III: Energy balance and flux. J. Phys. Oceanogr., 38, 2256-2269, doi:10.1175/ 2008JPO3810.1.

D'Asaro, E., C. Lee, L. Rainville, R. Harcourt, and L. Thomas, 2011: Enhanced turbulence and energy dissipation at ocean fronts. Science, 332, 318-322, doi:10.1126/science.1201515.

Ferrari, R., and C. Wunsch, 2009: Ocean circulation kinetic energy: Reservoirs, sources, and sinks. Annu. Rev. Fluid Mech., 41, 253-282, doi:10.1146/annurev.fluid.40.111406.102139. 
Garabato, A. N., K. L. Polzin, B. A. King, K. J. Heywood, and M. Visbeck, 2004: Widespread intense turbulent mixing in the Southern Ocean. Science, 303, 210-213, doi:10.1126/ science.1090929.

Garrett, C. J. R., and W. H. Munk, 1972: Space-time scales of internal waves. Geophys. Fluid Dyn., 3, 225-264, doi:10.1080/ 03091927208236082.

Grisouard, N., and L. N. Thomas, 2015: Energy exchanges between density fronts and near-inertial waves reflecting off the ocean surface. J. Phys. Oceanogr., 46, 501-516, doi:10.1175/ JPO-D-15-0072.1.

Jiang, J., Y. Lu, and W. Perrie, 2005: Estimating the energy flux from the wind to ocean inertial motions: The sensitivity to surface wind fields. Geophys. Res. Lett., 32, L15610, doi:10.1029/2005GL023289.

Large, W. G., J. C. McWilliams, and S. C. Doney, 1994: Oceanic vertical mixing: A review and a model with a nonlocal boundary layer parameterization. Rev. Geophys., 32, 363-403, doi:10.1029/94RG01872.

Leonard, A., 1974: Energy cascade in large-eddy simulations of turbulent fluid flows. Advances in Geophysics, Vol. 18, Academic Press, 237-248, doi:10.1016/S0065-2687(08)60464-1.

McWilliams, J. C., 2016: Submesoscale currents in the ocean. Proc. Roy. Soc. London, 472A, 20160117, doi:10.1098/rspa.2016.0117.

Molemaker, M. J., J. C. McWilliams, and I. Yavneh, 2005: Baroclinic instability and loss of balance. J. Phys. Oceanogr., 35, 1505-1517, doi:10.1175/JPO2770.1.

,-- - and X. Capet, 2010: Balanced and unbalanced routes to dissipation in an equilibrated Eady flow. J. Fluid Mech., 654, 35-63, doi:10.1017/S0022112009993272.

Munk, W. H., and E. Palmén, 1951: Note on the dynamics of the Antarctic Circumpolar Current. Tellus, 3, 53-55, doi:10.1111/ j.2153-3490.1951.tb00776.x.

Nagai, T., A. Tandon, E. Kunze, and A. Mahadevan, 2015: Spontaneous generation of near-inertial waves by the Kuroshio Front. J. Phys. Oceanogr., 45, 2381-2406, doi:10.1175/JPO-D-14-0086.1.

Nikurashin, M., G. K. Vallis, and A. Adcroft, 2013: Routes to energy dissipation for geostrophic flows in the Southern Ocean. Nat. Geosci., 6, 48-51, doi:10.1038/ngeo1657.

Phillips, H. E., and S. R. Rintoul, 2000: Eddy variability and energetics from direct current measurements in the Antarctic Circumpolar Current south of Australia. J. Phys. Oceanogr., 30, 3050-3076, doi:10.1175/1520-0485(2000)030<3050: EVAEFD $>2.0 . \mathrm{CO} ; 2$.

Ponte, A. L., and P. Klein, 2015: Incoherent signature of internal tides on sea level in idealized numerical simulations. Geophys. Res. Lett., 42, 1520-1526, doi:10.1002/2014GL062583.

Rimac, A., J.-S. Storch, C. Eden, and H. Haak, 2013: The influence of high-resolution wind stress field on the power input to nearinertial motions in the ocean. Geophys. Res. Lett., 40, 48824886, doi:10.1002/grl.50929.
Taylor, S., and D. Straub, 2016: Forced near-inertial motion and dissipation of low-frequency kinetic energy in a wind-driven channel flow. J. Phys. Oceanogr., 46, 79-93, doi:10.1175/ JPO-D-15-0060.1.

Thomas, L. N., 2012: On the effects of frontogenetic strain on symmetric instability and inertia-gravity waves. J. Fluid Mech., 711, 620-640, doi:10.1017/jfm.2012.416.

Tréguier, A.-M., and J. McWilliams, 1990: Topographic influences on wind-driven, stratified flow in a $\beta$-plane channel: An idealized model for the Antarctic Circumpolar Current. J. Phys. Oceanogr., 20, 321-343, doi:10.1175/1520-0485(1990)020<0321: TIOWDS $>2.0 . \mathrm{CO} ; 2$.

Ulloa, H. N., K. B. Winters, A. de la Fuente, and Y. Niño, 2015: Degeneration of internal Kelvin waves in a continuous two-layer stratification. J. Fluid Mech., 777, 68-96, doi:10.1017/jfm.2015.311.

Van Meurs, P., 1998: Interactions between near-inertial mixed layer currents and the mesoscale: The importance of spatial variabilities in the vorticity field. J. Phys. Oceanogr., 28, 1363-1388, doi:10.1175/1520-0485(1998)028<1363: IBNIML $>2.0 . \mathrm{CO} ; 2$.

Vanneste, J., 2013: Balance and spontaneous wave generation in geophysical flows. Annu. Rev. Fluid Mech., 45, 147-172, doi:10.1146/annurev-fluid-011212-140730.

Wagner, G., and W. Young, 2016: A three-component model for the coupled evolution of near-inertial waves, quasigeostrophic flow and the near-inertial second harmonic. J. Fluid Mech., 802, 806-837, doi:10.1017/jfm.2016.487.

Whitt, D. B., and L. N. Thomas, 2015: Resonant generation and energetics of wind-forced near-inertial motions in a geostrophic flow. J. Phys. Oceanogr., 45, 181-208, doi:10.1175/ JPO-D-14-0168.1.

Winters, K. B., 2015: Tidally driven mixing and dissipation in the stratified boundary layer above steep submarine topography. Geophys. Res. Lett., 42, 7123-7130, doi:10.1002/ 2015 GL064676.

_ , and A. de la Fuente, 2012: Modelling rotating stratified flows at laboratory-scale using spectrally-based DNS. Ocean Modell., 49-50, 47-59, doi:10.1016/j.ocemod.2012.04.001.

_ Boussinesq fluid flow. J. Fluid Mech., 714, 476-488, doi:10.1017/jfm.2012.493.

_ - P. Lombard, J. Riley, and E. A. D'Asaro, 1995: Available potential energy and mixing in density stratified fluids. J. Fluid Mech., 289, 115-128, doi:10.1017/S002211209500125X.

Xie, J. H., and J. Vanneste, 2015: A generalised-Lagrangian-mean model of the interactions between near-inertial waves and mean flow. J. Fluid Mech., 774, 143-169, doi:10.1017/ jfm.2015.251.

Young, W., and M. Ben-Jelloul, 1997: Propagation of near-inertial oscillations through a geostrophic flow. J. Mar. Res., 55, 735-766, doi:10.1357/0022240973224283. 